\title{
Pterobreathers in a model for a layered crystal with realistic potentials: Exact moving breathers in a moving frame
}

\author{
Juan F. R. Archilla $\odot,{ }^{1, *}$ Yusuke Doi, ${ }^{2}$ and Masayuki Kimura ${ }^{3}$ \\ ${ }^{1}$ Group of Nonlinear Physics, Universidad de Sevilla, ETSI Informática, Avda Reina Mercedes s/n, 41012-Sevilla, Spain \\ ${ }^{2}$ Department of Adaptive Machine Systems, Graduate School of Engineering, Osaka University, 2-1 Yamadaoka, Suita, \\ Osaka 565-0871, Japan \\ ${ }^{3}$ Department of Electrical Engineering, Kyoto University Katsura, Nishikyo-ku, Kyoto 615-8510, Japan
}

(Received 2 February 2019; published 12 August 2019)

\begin{abstract}
In this article we perform a thorough analysis of breathers in a one-dimensional model for a layered silicate for which there exists fossil and experimental evidence of moving excitations along the close-packed lines of the $\mathrm{K}^{+}$layers. Some of these excitations are likely breathers with a small energy of about $0.2 \mathrm{eV}$ as the numerically obtained breathers described in the present model. Moving breathers as exact solutions of the dynamical equations are obtained at the price of being generically associated with a plane wave, a wing, with finite amplitude, although this amplitude can be very small. We call them pterobreathers. For some frequencies the wings disappear and the solutions become exact moving breathers with no wings, showing the phenomenon of supertransmission of energy. We perform a theoretical analysis of pterobreathers in systems with substrate potential and show that they are characterized by a single frequency in the moving frame plus the frequency of the wings. We have also studied high-energy stationary breathers which transform into single and double kinks and stable multibreathers with very strong localization.
\end{abstract}

DOI: 10.1103/PhysRevE.100.022206

\section{INTRODUCTION}

Layered silicate muscovite mica long has been shown to have interesting properties. It was the second material for which nuclear tracks from fission fragments were detected [1] and also the first one for which fossil nuclear tracks were observed [2-4]. About the same time it was demonstrated that some dark tracks record the passage of swift particles as positrons, antimuons, and protons [5-7]. The tracks were produced by the precipitation of excess iron forming the mineral magnetite and growing by accretion in a similar way to a bubble chamber during some sensitive period with some specific conditions of pressure and temperature [7-9]. Swift particles could be produced by cosmic rays, neutrino interaction, and radioactive decay.

However, most of the dark tracks that appear in natural muscovite lacked the characteristic kinkiness of Coulomb scattering and were along the lattice directions in the cation plane sandwiched between tetrahedra-octahedra-tetrahedra silicate structures above and below. Therefore, they were proposed to be recording the passage of some lattice excitations that were named quodons [10,11]. A likely origin of some of the quodons is the recoil from beta decay of ${ }^{40} \mathrm{~K}$, the most abundant unstable isotope in potassium. Some of the recoils have the same momentum of the positrons emitted and recorded.

The propagation of localized energy along the lattice lines of the cation layer were demonstrated experimentally by

*archilla@us.es bombarding a sample with alpha particles and observing the ejection of an atom at the other side [12].

The different beta decays of ${ }^{40} \mathrm{~K}[13,14]$ have different recoil energies between 0 and $50 \mathrm{eV}$ and usually leave a change in the electric charge of the recoiling atom $[15,16]$. It was observed that only positive particles produced dark tracks $[17,18]$. For example, electrons from beta minus $\left(\beta^{-}\right)$ decay of ${ }^{40} \mathrm{~K}$, the dominant decay channel with $89 \%$ probability compared with $0.001 \%$ for beta plus $\left(\beta^{+}\right)$, were not recorded. However, the recoils of $\beta^{-}$decay have a positive charge and could trigger a localized excitation that can be recorded. The fact that the magnitude of dark decoration was similar for lattice excitations and for swift particles at nearly sonic speed, when they were about to stop, supported the conclusion that many of the lattice excitations also carry an electric charge $[16,18]$.

A new experiment demonstrated charge transport stimulated by nonlinear excitations produced by alpha bombardment in the absence of an electric field [19]. This phenomenon was dubbed hyperconductivity. Lately, charge transport in absence but also in presence of electric field has been observed in several different silicates [20].

While in previous publications, kinks and nanopterons have been studied in a model with realistic parameters [15, 21-24], there was not a systematic study of moving breathers in this model. Moving breathers are localized vibrational modes in nonlinear lattices [25]. Differently from kinks and nanopterons, moving breathers do not imply a permanent displacement and therefore mass transfer, which make them less energetic. They can also be seen as envelope solitons if their amplitude tends to zero at $\pm \infty$ [26]. Recently they have been shown to assist in energy transfer [27]. 
The existence of moving breathers in the model is of interest because in some mica crystals it can be seen that secondary nonlinear excitations are scattered from a primary one [28], and, therefore, they should have much smaller energies than energetic kinks. Some of them are shown to lose the electric charge but continue propagating and later recover the charge. These neutral energy carriers with low energy could be breathers. It has also been shown that the decay of ${ }^{40} \mathrm{~K}$ can lead to different types of perturbations, from kinks to breathers depending on the energies involved [16].

Moving breathers in models inspired by the tracks in muscovite have been found in one dimension [29] using a sinusoidal on-site potential and Morse interaction and in two dimensions [30-32] also using sinusoidal on-site potential and either piecewise or Lennard-Jones interaction potential. The lifetime of the moving breathers was large but no physical magnitudes as the energy were provided, making it difficult to relate with the experiments.

The differences with our present work are several. First, we use both an on-site potential and interaction potential obtained from the properties of the ions involved and the crystal structure and therefore being particularly appropriate and also providing values as energies, frequencies, or velocities in physical units. Second, instead of constructing approximate solutions, we construct exact solutions at the price of having wings, i.e., extended plane waves that do not decay in space or time. Exact solutions allow for continuation in the space of parameters and obtain many different ones. The existence of the wings implies that the solutions are not completely localized. However, the wings are often very small and even disappear for specific frequencies, providing a supertransmission channel [33].

We can also check the stability of the exact solutions through a variant of the Floquet analysis. Third, motivated by our results, we have developed a precise description of exact moving breathers, showing that they are described by a single frequency in the moving frame plus a related one for the wings. Both are integer multiples of some fundamental frequency.

Other interesting aspects of muscovite are its proposed use to detect dark matter [34,35], reconstructive transformations triggered by breathers [36-38], its supposed role in the origin of life [39,40], and the genetic code [41]. It has also been shown that thin layers behave as semiconductors and semimetals with many potential technological applications through bandgap engineering [42].

The article is organized in the following way: Sec. II describes the model with detail and the previous results. In Sec. III we review some breathers concepts, while in Sec. IV we specify some terminology and deduce approximate equations for the wings and tails in the model and compare with the numerical results. Sections V and VI describe stationary breathers and their energies and the observation of approximate small-amplitude moving breathers. In Sec. VII exact moving breathers are described mathematically for a given symmetry, and in Sec. VIII the plane waves with the same symmetry are considered, providing a extremely simple description of exact moving breathers in the moving frame. Section IX describes how the stability is calculated and provides some example of stable moving breathers. In Sec. X

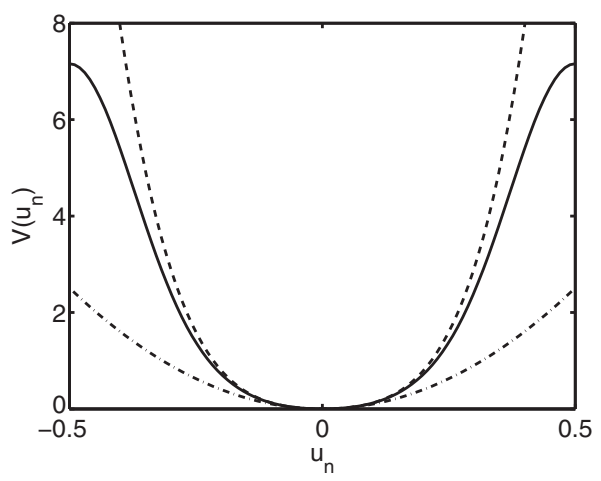

FIG. 1. Substrate potential given by Eq. (2) (continuous line), approximation to order 4 , i.e., $V\left(u_{n}\right)=\frac{1}{2} \omega_{0}^{2} u_{n}^{2}+\frac{1}{4} \beta u_{n}^{4}$ (dashed line) and harmonic potential (dashed-dotted line). Units of energy are $u_{E} \simeq 2.77 \mathrm{eV}$, and of distance, the lattice unit $u_{L}=5.19 \AA$.

some examples of moving breathers with wings are described, and in Sec. XI some moving breathers without wings are presented. Some interesting phenomena related with highenergy stationary breathers are commented on in Sec. XII. The article finishes with the conclusions

\section{MODEL AND PREVIOUS RESULTS}

The model we use has been proposed in Refs. [15,23]. It consists of a one-dimensional model for the rows of $\mathrm{K}^{+}$ions within the cation layers of muscovite mica.

The interaction potential is the sum of the electrostatic repulsion $\mathrm{k}_{\mathrm{e}} e^{2} / r$ plus a repulsive short-range potential obtained from the well-known ZBL potentials [43]. They are a sum of Yukawa-type potentials $\sum_{i=1}^{4} \gamma_{i} \mathrm{k}_{\mathrm{e}} Z^{2} e^{2} \exp \left(-r / \rho_{i}\right) / r$, with constants $\gamma_{i}$ and $\rho_{i}$ that are obtained from an universal function that depends only on the atomic number of the atoms involved. It represents the electrostatic repulsion between the $\mathrm{K}$ nuclei screened by the electrons in the atom or ion. ZBL potentials are used in ion bombardment modeling, and the four terms are necessary for different ranges of energies. For energies up to $100 \mathrm{keV}$ one term is enough, and thus only one term of the ZBL potential is kept.

The substrate potential was obtained as a sum of electrostatic interactions and empirical potentials used for silicates [44] considering the ions in several layers of the crystal at the equilibrium positions but excluding the $\mathrm{K}^{+}$, in the same row which are taken into account directly. The resulting substrate potential is a periodic potential which is well approximated by a Fourier series with four terms, and it is represented in Fig. 1. A peculiarity of it is that it is hard for small oscillations, i.e., the frequency increases with the amplitude, and soft for larger oscillations of $\gtrsim 0.3$ times the lattice unit. The substrate potential reproduces the experimental infrared frequency of $110 \mathrm{~cm}^{-1}$ for longitudinal vibrations in the potassium rows [45] and the $20 \mathrm{eV}$ height of the energy wells obtained by molecular dynamics [46].

We will use scaled units, which are, for distance, the equilibrium distance between ions $u_{L}=a=5.19 \AA$; for energy, the energy of interaction between ions at equilibrium, $u_{E}=\mathrm{k}_{\mathrm{e}} e^{2} / a \simeq 2.77 \mathrm{eV}$, where $\mathrm{k}_{\mathrm{e}}$ is the Coulomb constant and $e$ the elemental unit of charge; and for mass, 
the mass of a potassium atom $u_{M}=m_{K}=39.1 \mathrm{amu}$. Time becomes a derived unit with value $u_{T}=\left(u_{M} u_{L}^{2} / u_{E}\right)^{1 / 2}=$ $\left(m_{K} a^{3} / \mathrm{k}_{\mathrm{e}} e^{2}\right)^{1 / 2} \simeq 0.2 \mathrm{ps}$. The unit of frequency is therefore about $5 \mathrm{THz}$, and the unit of angular frequency is equivalent to $5 \mathrm{Trad} / \mathrm{s}$ or $1 /\left(2 \pi u_{T}\right) \simeq 0.8 \mathrm{THz} \simeq 26.7 \mathrm{~cm}^{-1}$.

In the scaled units, the Hamiltonian becomes

$$
H=\sum_{n}\left[\frac{1}{2} \dot{u}_{n}^{2}+V\left(u_{n}\right)+U\left(u_{n+1}-u_{n}\right)\right] \text {. }
$$

The substrate potential is given by

$$
V\left(u_{n}\right)=\sum_{m=0}^{4} v_{m} \cos \left(2 \pi m u_{n}\right)
$$

with $\quad\left\{v_{m}\right\}=[2.4474,-3.3490,1.0997,-0.2302,0.0321]$. The interaction potential is given by

$$
U\left(u_{n+1}-u_{n}\right)=\frac{1+B \mathrm{e}^{-\left|1+u_{n+1}-u_{n}\right| / \rho}}{\left|1+u_{n+1}-u_{n}\right|},
$$

with $B=184.1-510.79 \mathrm{eV}$ and $\rho=0.0569-0.29 \AA$.

Therefore the dynamical equations become

$$
\ddot{u}_{n}=-V^{\prime}\left(u_{n}\right)+U^{\prime}\left(u_{n+1}-u_{n}\right)-U^{\prime}\left(u_{n}-u_{n-1}\right) .
$$

The derivative of the substrate potential is given by

$$
-V^{\prime}\left(u_{n}\right)=\sum_{m=1}^{4} 2 \pi v_{m} \sin \left(2 \pi m u_{n}\right),
$$

and the interaction forces become

$$
\begin{array}{r}
U^{\prime}\left(u_{n+1}-u_{n}\right)=-\frac{1+B \mathrm{e}^{-\left|1+u_{n+1}-u_{n}\right| / \rho}}{\left(1+u_{n+1}-u_{n}\right)^{2}} \\
-\frac{B}{\rho} \frac{\mathrm{e}^{-\left|1+u_{n+1}-u_{n}\right| / \rho}}{\left|1+u_{n+1}-u_{n}\right|}
\end{array}
$$

and

$$
\begin{aligned}
-U^{\prime}\left(u_{n}-u_{n-1}\right)= & \frac{1+B \mathrm{e}^{-\left|1+u_{n}-u_{n-1}\right| / \rho}}{\left(1+u_{n}-u_{n-1}\right)^{2}} \\
& +\frac{B}{\rho} \frac{\mathrm{e}^{-\left|1+u_{n}-u_{n-1}\right| / \rho}}{\left|1+u_{n}-u_{n-1}\right|}
\end{aligned}
$$

\section{A. Nanopterons, supersonic kinks, or crowdions}

A supersonic crowdion or kink was described in Ref. [23] with an energy of $27 \mathrm{eV}$, which was coherent with the energy available in muscovite, a maximum of $42 \mathrm{eV}$ for the recoil from the $\beta^{-}$decay of ${ }^{40} \mathrm{~K}$ the main beta decay, which is thought the source of fossil tracks $[13,15]$ and larger than the 7-8 eV of energy to eject an atom, as shown experimentally [12]. Many different kinks or crowdions linked to a plane wave were found in Ref. [24], and many of them were stable. They are usually called nanopterons, but we think they are better named pterokinks as explained below. Also other unstable kinks without wings were found and stable double kinks or bicrowdions, some without wings. The latter are with energies still within the recoil energy available in some beta decays $[13,15,16]$.

\section{B. Small-amplitude moving breathers}

Also, it has been found stationary breathers with a broad range of energies and moving breathers with small energies $0.2-0.3 \mathrm{eV}$ that were produced by compressing a bond, observing the results, and refining the results [24]. The small energies of the moving breathers were coherent with the expected energies of secondary tracks in muscovite $[16,28]$.

\section{CONCEPTS ABOUT STATIONARY AND MOVING BREATHERS}

In this section we review some concepts of stationary and moving breathers that will be used later. Stationary discrete breathers, or just breathers for short, are periodic localized solutions of a discrete dynamical system with some kind of nonlinearity. They arise in a variety of systems of different dimensions, Hamiltonian, and dissipative. Here we will refer mainly to Klein-Gordon Hamiltonian systems, because it is the system of interest in this article. We will also restrict the notation to one dimension, although the generalization to several dimension is straightforward. The dynamical equations corresponding to a Klein-Gordon Hamiltonian system similar to Eq. (1) in scaled form is given by

$$
\mathcal{F}_{n}(u, \varepsilon)=\ddot{u}_{n}+V^{\prime}\left(u_{n}\right)+\varepsilon \frac{\partial W(u)}{\partial u_{n}}=0,
$$

where $u=\left\{u_{n}(t)\right\}$, and $u_{n}$ are the variables measured with respect to their equilibrium positions. $V\left(u_{n}\right)$ is the substrate potential, with a minimum at $u_{n}=0$, i.e., $\omega_{0}^{2}=\partial^{2} V(0) / \partial u_{n}^{2}>$ $0, \omega_{0}$ being the oscillation frequency of the isolated oscillators in the limit of small amplitude. The coupling potential is represented by $W(u)=\sum_{n} U\left(u_{n+1}-u_{n}\right)$ and $\varepsilon$ is a parameter to modulate the strength of the coupling. Any solution can be characterized by the initial variables and momenta or by the Fourier coefficients up to some order if it is a periodic solution.

At $\varepsilon=0$, the system is said to be at the anticontinuous limit [47] and consists of isolated oscillators with the same dynamical equation:

$$
\ddot{u}_{n}+V^{\prime}\left(u_{n}\right)=0 .
$$

This equation has a periodic solution $u_{n}^{0}$ of frequency $\omega_{b}$ that depends on the amplitude of oscillation $A$, i.e., $\omega_{b}=\omega_{b}(A)$. If $\omega_{b}>\omega_{0}$ the potential $V$ is called hard and soft otherwise. Equation (9) also has two other solutions: one is the trivial solution $u_{n}=0$ with frequency $\omega_{b}$, and the other is the solution with very small amplitude and frequency $\omega_{0}$. Therefore, there exists at $\varepsilon=0$ a periodic solution $u(0)$ completely localized in one single site with a single oscillator with frequency $\omega_{b}$ and amplitude $A\left(\omega_{b}\right)$ and the rest of them with zero amplitude but the same frequency.

The solution $u(0)$ can be continued to $u(\varepsilon)$ for $\varepsilon>0$ provided that there is no other branch of solutions in the vicinity of $u(0)$, which is obtaining by restricting the space of solutions, for example, to time-invariant solutions. There are two conditions for the implicit function theorem to be applied, (a) that neither $\omega_{b}$ nor its harmonics $n \omega_{b}$ are inside the phonon band (which is just $\omega_{0}$ at $\varepsilon=0$ ) and (b) that $\partial \omega_{b} / \partial A \neq 0$, i.e., that the system is truly nonlinear at that frequency $[47,48]$. The amplitudes of the solution $u(\varepsilon)$ decay 
exponentially around the initial excited site for short-range coupling. Equally, it is possible to prove the existence of localized multibreathers when several sites are excited at the anticontinuous limit. In practice, the solution can often be continued for some range of frequencies up to $\varepsilon=1$ reobtaining the original system.

Note that stationary breathers or breathers at rest have a single frequency $\omega_{b}$, which is related to the rest breather frequency defined in Sec. VIII E.

The operator $\mathcal{N}=\partial \mathcal{F} / \partial u$ is called the Newton operator, which acts on the perturbation $\xi=\left\{\xi_{n}\right\}$ of $u$ as

$$
\mathcal{N}_{n}(u, \varepsilon) \xi=\ddot{\xi}_{n}+V^{\prime \prime}(u) \xi_{n}+\varepsilon \sum_{m} \frac{\partial^{2} W(u)}{\partial u_{n} \partial u_{m}} \xi_{m} .
$$

The solutions of $\mathcal{N}(u, \varepsilon) \xi=0$ give the evolution of the perturbation of a solution $u$ of $\mathcal{F}(u)=0$ given by $u+\xi$ in the linear approximation. The eigenvalues of the equation $\mathcal{N} \xi=E \xi$ form bands that are used to determine breather or multibreather stability [48,49].

The theory can be extended to nonhomogeneous on-site potentials, or with several minima, to FPU systems, that is, without on-site potential, where different proofs have to be applied [50,51]. It can also be extended to many other models, for example, systems with long-range interaction with different geometries [52,53] and many others.

Stationary breathers can be put into movement by some perturbation of the velocities that break the symmetry of the breather, and a simple method is the discrete gradient method, i.e., $v_{n}=\lambda\left(u_{n+1}-u_{n-1}\right)$, for some small $\lambda$ [52]. The best mobility is obtained with the pinning mode method, although it is valid only for specific values of the coupling constant or specific frequencies, for which two specific eigenvectors appear [54]. Of course, there are many other approximate methods that use different ansatzes $[27,55]$.

However, breathers obtained by these procedures are not exact solutions of the dynamical equations. They have a continuous spectrum of frequencies and radiate small-amplitude waves and therefore have a finite lifetime.

The frequency $\omega_{b}$ of the stationary breather which has been perturbed no longer characterizes the moving breather, although it is often used to parametrize moving breathers.

We will construct exact solutions called pterobreathers in Sec. VII at the price of having wings (see below), but for some specific frequencies, the wings disappear, and we will obtain exact moving breathers.

\section{PHONONS, TAILS, WINGS, AND TERMINOLOGY}

In this section we will define some terms and concepts that we think are convenient with respect to the smaller amplitude waves accompanying a solitary wave sometimes called tails. We will also apply these concepts to our specific system of interest.

There are two different concepts, tails and wings. A tail is the perturbation of the particles at the borders of the core of a solitary wave, as a breather, soliton, or kink, while a wing is an extended solution that does not decay in space or time that accompanies those solitary waves. Wings are very important in this system and in most systems with a substrate potential, therefore we will clarify the concepts in this section and will be precise in the terminology.

Exact traveling solutions will be parameterized by some (rest) breather frequency $\omega_{b}$ and parameters step $r$ and $m_{b}$. The traveling solution reproduces its profile displaced $r$ sites after $m_{b}$ internal oscillations. The frequency $\omega_{b}$ is the frequency of the stationary breather, whose perturbation becomes a traveling solution. These concepts will be defined with precision in Secs. VII and VIII.

\section{A. Tails and wings}

Tails and wings have one thing in common, which is that they have a smaller amplitude than the breather core, and therefore it is possible an approximate description supposing that the perturbations are small and the Taylor expansion to degree one, i.e., the linearized equation, or to some order is approximately valid. This reasoning is very useful to get insight into the properties of the solutions of the full system. As tails are behind the body in animals that move forward, the proper tail would hang behind a solitary wave, with diminishing amplitude. However, we will use tail also for the front tail, which can be much steeper. Very often back tails are taking energy from the solitary wave and making it smaller and slower and eventually bringing it to a stop [21,22].

\section{B. Solitary waves with wings}

Wings, however, are part of the solution, which does not exist without them. Thus, they allow the solitary wave to exist and to fly and are located equally at both sides, in front and behind. With periodic boundary conditions, they are the same wing. Kinks with wings are often called nanopterons, i.e., with dwarf wings, from the Greek. This term is, however, problematic for two reasons. First, the modern use of the prefix nano as $10^{-9}$ and its subsequent use in technology suggests something extremely small with respect to the solitary wave $\left(\sim 10^{-9}\right)$, which is not correct. Wings are in general smaller than the solitary-wave core but not that small. Second, it does not specify to what entity the wings are attached to. Therefore we propose the terms pterokink, pterosoliton, and pterobreather, but we will use also the synonyms winged-kinks, kinks with wings, and similar terms. More clarification will be issued with the specific system we are interested in.

\section{Approximate dynamical equations}

If we suppose that the perturbations are relatively small, we can expand Eq. (4) as a Taylor series. As the force due to the substrate potential is an odd function, the first nonlinear term is of order 3. The dynamical equations up to order 3 are

$$
\begin{aligned}
\ddot{u}_{n}= & -\omega_{0}^{2} u_{n}-\beta u_{n}^{3}+c_{s}^{2}\left[\left(u_{n+1}-u_{n}\right)-\left(u_{n}-u_{n-1}\right)\right] \\
& -3\left[\left(u_{n+1}-u_{n}\right)^{2}-\left(u_{n}-u_{n-1}\right)^{2}\right]+4\left[\left(u_{n+1}-u_{n}\right)^{3}\right. \\
& \left.-\left(u_{n}-u_{n-1}\right)^{3}\right]
\end{aligned}
$$

with $\quad \omega_{0}^{2}=-\sum_{m=1}^{4} v_{m}(2 \pi m)^{2}=4.48^{2}, \beta=$ $\sum_{m=1}^{4} v_{m}(2 \pi m)^{4} / 3 \simeq 991.650, \quad$ and $\quad c_{s}^{2}=2$. The corresponding substrate and interaction potentials become, respectively, $V\left(u_{n}\right)=\frac{1}{2} \omega_{0}^{2} u_{n}^{2}+\frac{1}{4} \beta u_{n}^{4}$, known as the $\phi^{4}$ potential, and $U\left(u_{n+1}-u_{n}\right)=\sum_{m=1}^{4}(-1)^{m}\left(u_{n+1}-u_{n}\right)^{m}$. 


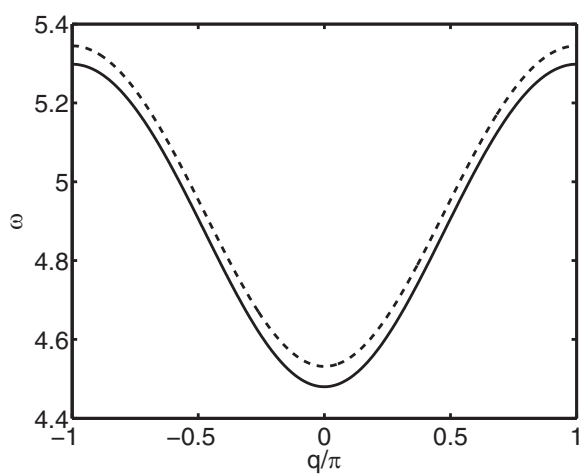

FIG. 2. Dispersion relation for phonons, given by Eq. (13) (continuous line) and for nonlinear plane waves or wings (dashed line) given by Eq. (15) for an amplitude $A=0.025$, which gives a frequency $\omega_{w}=5.35$ for $q=\pi$ similar to the one of the moving breathers found in this article and larger than their wings. The unit of angular frequency corresponds to $\simeq 0.8 \mathrm{THz}$.

As can be seen in Fig. 1 the $\phi^{4}$ potential is a good approximation for values of amplitudes around 0.1 . The order 4 approximation of $U$ is even better. Actually for those amplitudes the harmonic approximation of $U$ is quite good.

\section{Phonons}

Keeping only the linear terms in Eq. (11) we obtain the linearized dynamical equations

$$
\ddot{u}_{n}=-\omega_{0}^{2} u_{n}+c_{s}^{2}\left[u_{n+1}+u_{n-1}-2 u_{n}\right],
$$

with solutions $u_{n}=\exp \left(i\left[q n-\omega_{L} t\right)\right.$ and dispersion relation (DR)

$$
\omega_{L}^{2}=\omega_{0}^{2}+4 c_{s}^{2} \sin ^{2}\left(\frac{q}{2}\right)
$$

which is represented in Fig. 2. The subindex $L$ in the frequency reminds us that this is the laboratory frequency of the phonon with wave number $q$. This distinction will become important.
This dispersion relation is optical, i.e., bounded from below by $\omega_{0}$ at $q=0$. It is also bounded from above by $\omega_{\text {top }}=\left(\omega_{0}^{2}+\right.$ $\left.4 c_{s}^{2}\right)^{1 / 2} \simeq 5.298$ at $q=\pi$. The phase velocities $V=\omega / q$ tend to $\infty$ when $q \rightarrow 0$ and are bounded from below in the first Brillouin zone by $V_{\mathrm{ph} \text {, } \min }=\omega_{\mathrm{top}} / \pi \simeq 1.686$.

The sound velocity $c_{s}$ for the system without substrate is defined as the velocity when $q \rightarrow 0$, and it is both the maximum phase and group velocity. However, for a system with an optical dispersion relation, the sound velocity it is better defined as the maximum group velocity $V_{g}=d \omega_{L} / d q=$ $c_{s}^{2} \sin (q) / \omega_{L}$, which is $V_{g, \max } \simeq 0.4091$ at $q=0.4733 \pi$.

Note that $V_{g}=0$ at $q=0$ and $q= \pm \pi$. The mode $q=$ 0 is obviously at rest, but also the mode $q=\pi$ becomes $u_{n}=(-1)^{n} \exp \left(-i \omega_{\text {top }} t\right)$, which actually represents an outof-phase vibration that does not travel. In both cases the group velocity is also zero. These remarks will be of interest for the description of the moving breathers because stationary breathers will be a localized nonlinear version of these two modes for soft and hard substrate potentials [27], respectively. Stationary breathers are often also the starting point for calculation of exact moving breathers. In our system with a hard substrate potential the interesting one is only the mode $q=\pi$.

\section{E. Wings as nonlinear plane waves}

Wings are extended traveling waves that accompany the solitary wave. Far enough from the core and supposing their amplitude is relatively small, we can suppose that because they are extended, they have a single value of the wave vector, that is, they are given approximately by a function $u_{n}=$ $A \cos \left(q n-\omega_{w} t+\phi_{0}\right)$. It is obvious that it is not possible to add a constant term due to the form of the substrate potential. The linear equations are without interest because they will just lead to phonons, so we have to keep the approximation given by Eq. (11).

If we denote $\phi=q n+\phi_{0}$, then $u_{n}=A \cos (\phi), u_{n \pm 1}=$ $A \cos (\phi \pm q)$, and using elementary trigonometric relations we can express the different terms in Eq. (11) as functions of the harmonics of $u_{n}$, that is,

$$
\begin{aligned}
& u_{n}=A \cos (\phi) ; \quad \ddot{u}_{n}=-\omega_{w}^{2} \cos (\phi) ; \quad u_{n}^{3}=A^{3}\left[\frac{3}{4} \cos (\phi)+\frac{1}{4} \cos (3 \phi)\right] ; \quad\left(u_{n \pm 1}-u_{n}\right)=\mp 2 A \sin \left(\phi \pm \frac{q}{2}\right) \sin \left(\frac{q}{2}\right) \\
& {\left[\left(u_{n+1}-u_{n}\right)-\left(u_{n}-u_{n-1}\right)\right]=-2 A \sin ^{2}\left(\frac{q}{2}\right) \cos (\phi) ; \quad\left[\left(u_{n+1}-u_{n}\right)^{2}-\left(u_{n}-u_{n-1}\right)^{2}\right]=4 A^{2} \sin ^{2}\left(\frac{q}{2}\right) \sin (q) \sin (2 \phi)} \\
& {\left[\left(u_{n+1}-u_{n}\right)^{3}-\left(u_{n}-u_{n-1}\right)^{3}\right]=-4 A^{3} \sin ^{3}\left(\frac{q}{2}\right)\left[3 \cos (\phi) \sin \left(\frac{q}{2}\right)-\cos (3 \phi) \sin \left(\frac{3 q}{2}\right)\right]}
\end{aligned}
$$

Substitution of the above equation in Eq. (11) and collecting together the different harmonics we can see that the proposed solution is not possible because the harmonics above the first do not cancel out. More harmonics are necessary for an exact solution. Nonetheless the coefficients of the first harmonic have to be zero, which is called the rotating wave approximation, leading to the equation

$$
\omega_{w}^{2}=\omega_{0}^{2}+4 c_{s}^{2} \sin ^{2}\left(\frac{q}{2}\right)+\left[\frac{3}{4} \beta+48 \sin ^{4}\left(\frac{q}{2}\right)\right] A^{2} .
$$

The second term on the coefficient of $A^{2}$ changes by only about $2 \%$ the value of the correction to the phonon band. Therefore the nonlinear plane waves have frequencies above the phonons with the same wave number given approximately by $\omega_{w}^{2} \simeq \omega_{p h}^{2}+\frac{3}{4} \beta A^{2}$. They are shown in Fig. 2 .

It will be shown in Sec. VII that the wing frequency can be obtained as a function of the breather frequency in the limit of zero amplitude, what we will call the resonant phonon. We can measure the amplitude of the wings as a function of the breather frequency in numerical simulations 

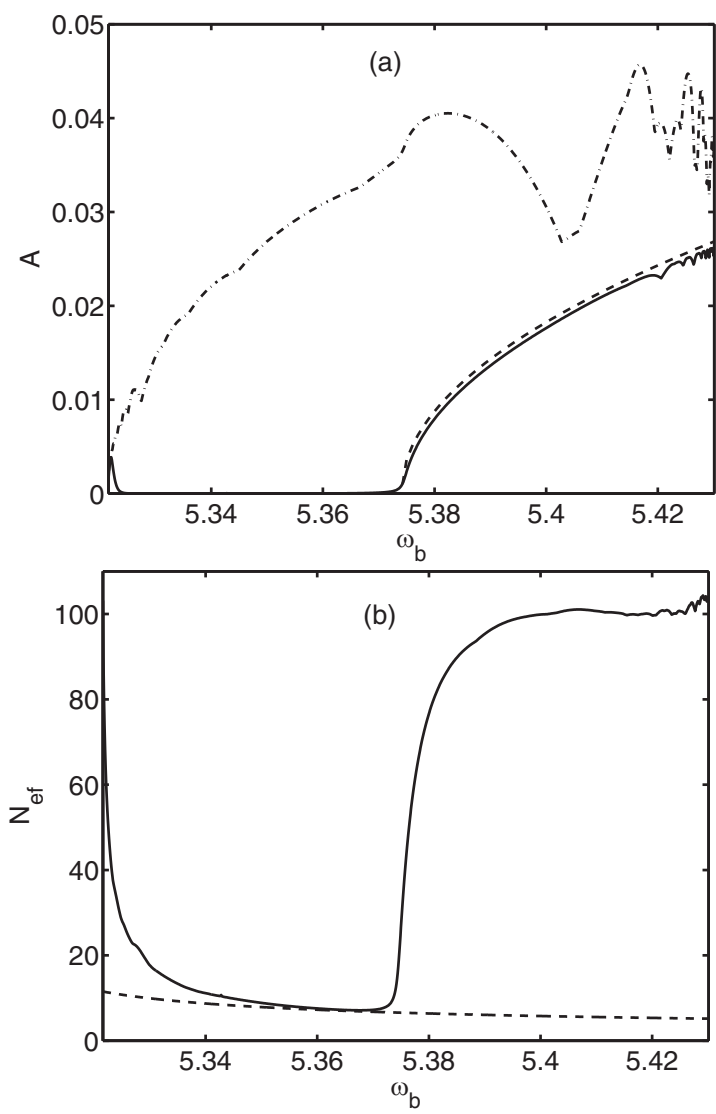

FIG. 3. (a) Amplitude of the wings obtained numerically (continuous line) compared with the wing amplitudes obtained with the rotating wave approximation from Eq. (15) (dashed line), using $\omega_{w}=\omega_{b}-\Delta \omega$, with $\Delta \omega=0.584$, the shift between the breather frequency and the wing frequency. Also is represented the amplitude of the solitary wave $\left(\max \left|u_{n}\right|\right)$ (dot-dashed line). (b) Measuring localization with the function effective number $N_{\text {eff }}$ for the numerical exact solutions (continuous line) and theoretically (dashed line). The theoretical one is good only when the solutions become localized and with small wings. Lattice with $N=128$ particles. (Both) Traveling solutions with parameters $r=1$ and $m_{b}=6$. The unit of distance is the lattice unit $5.19 \AA$, and the unit of angular frequency corresponds to $0.8 \mathrm{THz}$.

and compare it with the results of applying Eq. (15) isolating $A$ as a function of the frequency. The wing frequency depends on $\omega_{b}$, but if we suppose that in the first approximation the shift $\Delta \omega=\omega_{b}-\omega_{w}$ is constant for a small interval and equal to the observed value near the values where the wings disappear, $\Delta \omega=0.584$, we can obtain $A=A\left(\omega_{b}\right)$ from Eq. (15). Figure 3(a) shows the good agreement in spite of the different characteristics of the solitary waves involved, which appear in the irregular behavior of the solitary-waves amplitude.

\section{F. Tails}

Tails will be defined here as a part of the perturbation with amplitude small enough so as that the linear approximation holds and they are at the borders of a traveling solitary wave with larger amplitude with velocity $V_{b}$.
Mathematically we can approximate them with an equation of the form

$$
u_{n}(t)=A \exp \left(-\xi\left|n-V_{b} t\right|\right) \exp \left(i\left[q n-\omega_{L} t\right]\right)
$$

where $\xi$ is the inverse of the decay length $\Lambda$. We can use the complex form, because the equations we are using are linear. The equation will be valid for $n>V_{b} t$ or for $n<V_{b} t$ changing $\xi$ to $-\xi$. Substitution of $u_{n}$ in Eq. (12) and collecting together real and imaginary parts, the following equations are obtained:

$$
\begin{aligned}
\omega_{L} V_{b} \xi & =c_{s}^{2} \sinh (\xi) \sin (q) \\
\omega_{L}^{2} & =\xi^{2} V_{b}^{2}+\omega_{0}^{2}+2 c_{s}^{2}[1-\cos (\xi) \cos (q)] .
\end{aligned}
$$

If $V_{b}=0$, either $\xi=0$ and we recover a phonon, or $q=0$ or $\pm \pi$. For hard potentials, the breather is derived from the $\pi$ mode. So we can obtain an approximation to the frequency of the breather at rest as

$$
\omega_{b}^{2} \sim \omega_{0}^{2}+4 c_{s}^{2}+4 c_{s}^{2} \sinh ^{2}\left(\frac{\xi}{2}\right),
$$

that is, $\omega_{b}^{2}$ is above the top of the phonon band $\omega_{\text {top }}^{2}=\omega_{0}^{2}+$ $4 c_{s}^{2}$ in $4 c_{s}^{2} \sinh ^{2}(\xi / 2)$. For a typical moving breather with frequency $\omega_{b}=5.35, \xi=0.52$ meaning a change of $60 \%$ between neighboring particles.

We can measure the localization with the function effective number, defined for a set a numbers $\left\{A_{m}\right\}$ as $N_{\text {eff }}(A)=$ $\left(\sum\left|A_{m}\right|\right)^{2} /\left(\sum\left|A_{m}\right|^{2}\right)$. The effective number of the tail in Eq. (16) centered at $n_{0}=N / 2+0.5$ can be calculated, $N_{\text {eff }}\left(\left\{u_{n}\right\}\right)=2 \tanh (N \xi / 4) / \tanh (\xi / 2)$, but as the tanh of a few argument units is very close to 1 and keeping only the first term on $\xi / 2$, this expression is very similar to simply $N_{\text {eff }}(\xi) \simeq 4 / \xi$. In this way, we can obtain $\xi\left(\omega_{b}\right)$ from Eq. (19) and construct the function $N_{\text {eff }}\left(\omega_{b}\right)$.

We can compare this theoretical and approximate $N_{\text {eff }}$ with the one obtained with the numerical results for exact solutions. We should expect this approximation to fail when the wings are large or the solutions are not localized, as can be seen in Fig. 3(b). The simple approximation for the tails cannot reproduce the full richness of the dynamics, but it is quite good when the solutions are localized and the wings become small. Figure 4 compares the tail approximation with an exact numerical solution. It is also useful to visualize the difference between wings and tails.

\section{STATIONARY BREATHERS WITH SMALL AMPLITUDE}

Stationary breathers of the Sievers-Takeno (ST) type or particle-centered breathers and of the Page type $(\mathrm{P})$ or bondcentered breathers exist in the system. Both have an staggered profile and frequencies above the phonon spectrum. The ST breathers are linearly stable, and the $\mathrm{P}$ breathers are unstable. For the same frequency the latter have more energy at higher frequencies, although they tend to the same value $E_{\text {lim }} \simeq$ $0.29 \mathrm{eV}$ at a lower frequency $\omega \simeq 1.2 \omega_{0}$ close to the top of the phonon band. The energies are plotted in Fig. 5. Therefore, it is expected that moving breathers could appear when the Peierls-Nabarro barrier [56], that is, the difference between the energies of the two types of breathers, vanishes or is very small. 


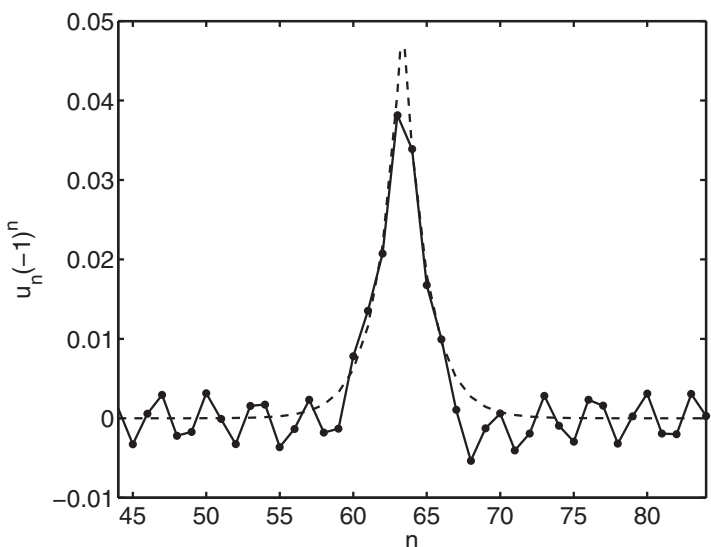

FIG. 4. Plot of the displacements multiplied by $(-1)^{n}$ for a breather with wings (black circles joined by a continuous line) and the approximate tail amplitudes $u_{n}=A \exp \left(-\xi\left|n-n_{0}\right|\right)$. The localization parameter $\xi$ is obtained theoretically from Eq. (19) (dashed line). The exact solution has been obtained in the full system with parameters $r=1, m_{b}=6$, and frequency $\omega_{b}=5.3755$ giving $\xi=0.63$. The tail approximation is very good, especially at the front but does not include the wings, which do not correspond to the tail trial solution. It can also be observed that the breather core has an wave number close to $q=\pi$ but the wings have a very different one. The effective number of the tail is $N_{\text {eff }}(\xi) \simeq 6.5$, very different from $N_{\text {eff }}\left(\left\{u_{n}\right\}\right) \simeq 36$ due to the wings. The unit of distance is the lattice unit $5.19 \AA$.

\section{APPROXIMATE SMALL-AMPLITUDE MOVING BREATHERS}

Different perturbations such as a local compression can lead to irregular stationary breathers and the ejection of one or more moving breathers of small amplitude. It is possible to obtain the coordinates and velocities of the moving breathers for a few particles and restart the simulation in an unperturbed lattice.

In this way a moving breather with good propagation properties is obtained. It is still far from an exact moving

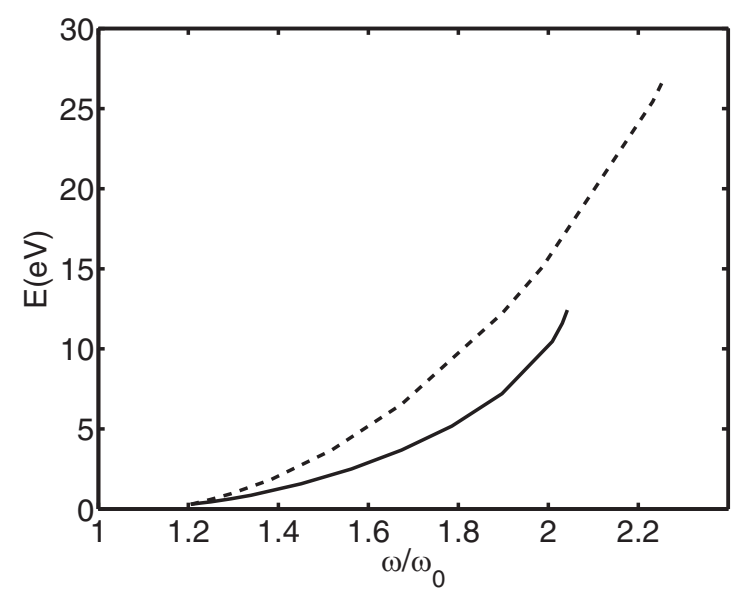

FIG. 5. Energies of the two types of stationary breathers, particle-centered or Sievers-Takeno breather (continuous line) and bond-centered or Page breather (dashed line). The latter is unstable.

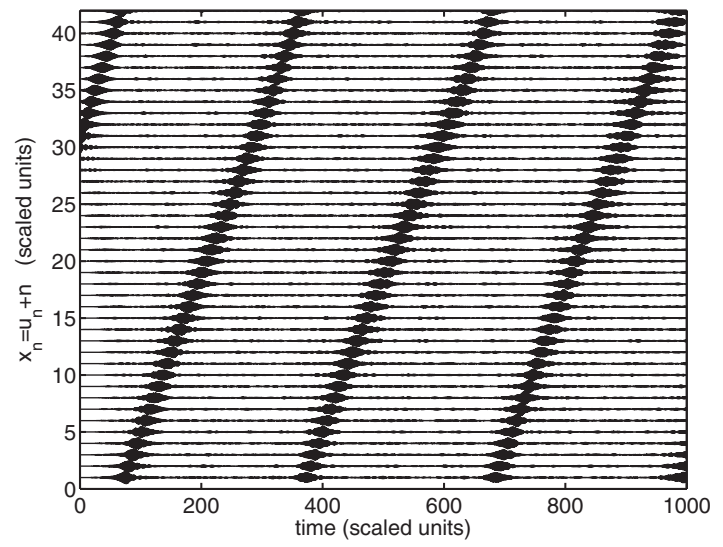

FIG. 6. Propagation of a moving breather emitted by a perturbed stationary breather. It can be seen that it emits phonons and that its velocity diminishes. The initial conditions obtained after extracting five positions and velocities are $u_{0}(29: 33)=\{0.0183,-0.0501,0.0370,-0.0106,0.0011\} \quad$ and $p_{0}(29: 33)=\{0.0692,0.0056,-0.1636,0.1448,-0.0848\} \quad$ with velocity $\simeq 0.14 \simeq 370 \mathrm{~m} / \mathrm{s}$ and energy of $0.0901 \simeq 0.25 \mathrm{eV}$. The unit of time is $\simeq 0.2$ ps and of distance, the lattice unit $5.19 \AA$.

breather as it mixed with phonons and its velocities change slightly with time. The propagation of this type of breather is represented in Fig. 6. They are also useful as a seed to obtain exact moving breathers.

By performing the two-dimensional discrete Fourier transform of the coordinates $u_{n}(t)$ it is possible to represent the breather in $\omega-q$ space as can be seen in Fig. 7. The intensity in the vicinity of several points of the phonon dispersion relation shows very clearly the presence of abundant phonons. Note that some intensity appears near the intersection of the sloped line corresponding to the breather with the linear phonon band. It corresponds to the wings.

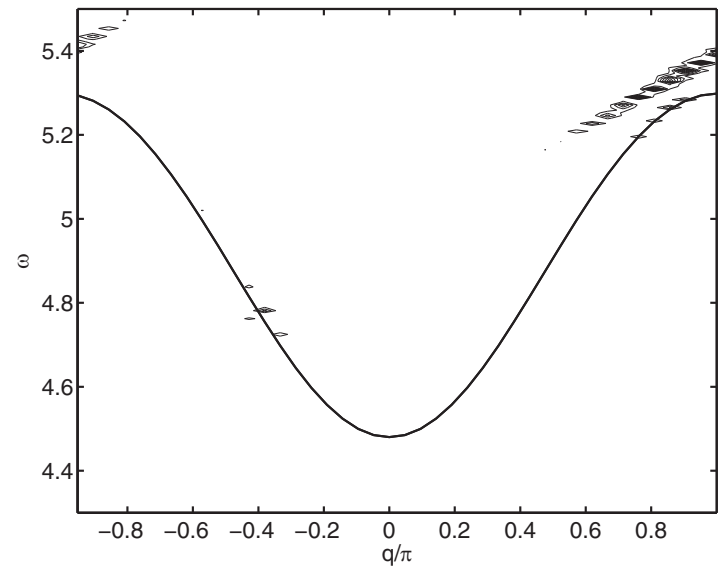

FIG. 7. Contour plot of the 2D DFT representation of the moving breather in Fig. 6 in $\omega-q$ space. The breather is the straight line centered at $q \simeq 0.9 \pi$ and $\omega \simeq 5.34$, the carrier wave vector and frequency, respectively. The continuous black line represents the calculated phonon dispersion relation. Some phonons can be seen around $q \simeq 0.9 \pi$ and a resonance of the moving breather near $q \simeq$ $-0.4 \pi$. The large hole in the breather intensity suggests that for other frequencies a nonresonant breather might be obtained. The unit of angular frequency corresponds to $0.8 \mathrm{THz}$. 


\section{PTEROBRETHERS: EXACT MOVING BREATHERS WITH WINGS}

Exact traveling solutions can be constructed using the Newton method from a good seed [57], such as the one obtained in Sec. VI. We propose a definition for an exact moving breather, with or without wings, as a solution $u=\left\{u_{n}\right\}$ of the dynamical system with the following three properties, which will be explained below: (1) it is an exact traveling solution, (2) it is localized, and (3) it has an internal frequency.

(1) It is an exact traveling solution, that is, it reproduces itself after a time $T_{F}$ but displaced an integer distance, the step $r$. It is a fixed point of the map:

$$
\hat{S} \equiv \hat{L}^{r} \hat{T}: \quad\left\{u_{n}(t)\right\} \rightarrow\left\{u_{n+r}\left(t+T_{F}\right)\right\}
$$

where $\hat{L}$ is the lattice unit translation operation and $\hat{T}$ is the time evolution map for the time $T_{F}$.

We will call $T_{F}$ the fundamental time or period of the moving breather. The time $T_{F}$ and integer $r$ are the minimum values with the invariance property above. It is clear that $u$ is also a fixed point of $\hat{S}^{m}$, for any integer $m$, which is a similar map with time $m T_{F}$ and integer step $m r$.

The frequency $\omega_{F}=2 \pi / T_{F}$ will be called the fundamental frequency of the moving breather.

The velocity of translation is $V_{b}=r a / T_{F}$, for $a$ the lattice distance. If we take $a$ as the unit of length,

$$
V_{b}=\frac{r}{T_{F}} \quad \text { and } \quad \omega_{F}=\frac{2 \pi}{T_{F}} .
$$

The time $T_{V}=1 / V_{b}$ for the MB to travel a unit distance is therefore $T_{V}=T_{F} / r$, i.e., the fundamental period $T_{F}$ is an integer multiple of $T_{V}$ :

$$
T_{F}=r T_{V} \equiv \frac{r}{V_{b}} .
$$

The concept of fundamental time or period was called propagation time in Ref. [58] and simply period in Ref. [59] for solitons. It avoids defining exact moving breathers in terms of an ill-defined breather frequency, which is a clear concept only for stationary breathers. However, the precise meaning for several breather frequencies will be established below.

(2) It is localized, that is, it is possible to write it as a function (or a sum of functions) of the form

$$
u_{n}(t)=f\left(n-V_{b} t, \omega_{i} t\right),
$$

where $f\left(\cdot, \omega_{i} t\right)$ is an at least partially localized function of the first argument $z=n-V_{b} t$ and a $2 \pi$ periodic function of the second argument $\Phi_{i}=\omega_{i} t$. The frequency $\omega_{i}$ is an internal frequency of the moving breather, that is, a frequency measured in the moving frame with velocity $V_{b}$ that accompanies the MB.

If $f(z, \phi) \rightarrow 0$ when $z \rightarrow \pm \infty$ and $\omega_{i}=0$, the solution is a soliton. If it tends to 0 only in $-\infty(+\infty)$ and to some finite value at $+\infty(-\infty)$ it is a kink (antikink).

(3) It has an internal vibration, that is $\omega_{i} \neq 0$.

The solution $u$ will correspond to an exact moving breather or sliding breather if $f \rightarrow 0$ when $z \rightarrow \pm \infty$.

However, it is a feature observed numerically that exact moving breather solutions that tend to zero at $n \rightarrow \pm \infty$ are exceptions for some specific potentials. In Ref. [60] an interaction potential was constructed that supported exact breathers both without a substrate potential and with a harmonic one. In general, the solution tends to a finite nonzero value, although frequently this is small or very small.

The same problem happens with kinks [24], the reason for it being that the phonon spectrum in a K-G system is optical, i.e., bounded from below, and therefore, the phase velocity of phonons is not bounded from above. It is bounded from below in the first Brillouin zone but not in the extended zone.

It is easy to obtain the conditions for Eq. (23) to be invariant with respect to the map in Eq. (20):

$$
\begin{aligned}
f & {\left[n+r-V_{b}\left(t+T_{F}\right), \omega_{i}\left(t+T_{F}\right)\right] } \\
& =f\left[n-V_{b} t+\left(r-V_{b} T_{F}\right), \omega_{i} t+\omega_{i} T_{F}\right] \\
& =f\left(n-V_{b} t, \omega_{i} t+\omega_{i} T_{F}\right)=f\left(n-V_{b} t, \omega_{i} t\right) .
\end{aligned}
$$

The first arguments repeats itself because $r-V_{b} T_{F}=0$, due to the definition of $T_{F}$ and as the second argument is $2 \pi$ periodic, the condition for $u$ to be invariant under $\hat{S}$ is $\omega_{i} T_{F}=$ $2 \pi m$ or $\omega_{i}=m\left(2 \pi / T_{F}\right)$ :

$$
\omega_{i}=m \omega_{F} .
$$

In other words, the internal frequencies of a MB are integer multiples of the fundamental frequency $\omega_{F}$.

The general solution will be of the form

$$
u_{n}(t)=\sum_{m=-\infty}^{m=\infty} f_{m}\left(n-V_{b} t, m \omega_{F} t\right),
$$

although in practice, only very few $m$ terms are significant, basically two, as we will see below.

In this way, Eq. (23) can be written as $f=g+h$, where $g$ tends to zero at $\pm \infty$ and $h$ corresponds to the wings, i.e., a relatively small nonlinear extended wave, or, in some cases, quasilinear. Neither $g$ nor $h$ is an exact solution of the nonlinear dynamical solution by themselves, but both $g$ and $h$ are fixed points of $\hat{S}$ and therefore have the same symmetry $\hat{S}$. As stated before, we prefer the term wing for $h$ than tail. Tails correspond to nonexact, and therefore, transient moving breather solutions which leave the debris of a tail behind it [61].

Note that $m \omega_{F}$ are the frequencies in the moving frame and not in the laboratory frame, where the discrete Fourier transform (DFT) operates, and it is used to obtain the $\omega-q$ representation of the MB [33].

For example, in one of the exact breathers found in the present system, the breather advances two sites (step $r=2$ ) in the fundamental time $T_{F}=14.1442$ with a fundamental frequency $\omega_{F}=0.4442, V_{b}=r / T_{F}=0.1414$.

\section{RESONANT PLANE WAVES}

Plane waves are given by the equation

$$
u_{n}(t)=A \exp \left(i\left[q n-\omega_{L} t\right]\right)
$$

where $A$ is the amplitude, $q$ is the wave number, and $\omega_{L}$ the laboratory frequency. They can be solution of the linearized equation Eq. (12), in which they are phonons and their dispersion relation is given by Eq. (13). 
But plane waves are also a basis for the function space $\left\{f_{n}(t)\right\}_{n=1}^{N}$ with periodic boundary conditions and therefore with wave numbers $q_{l}=2 \pi l / N$ for $l=0, \ldots, N-1$, that is,

$$
\begin{aligned}
f_{n}(t) & =\sum_{l} \int_{t} A_{l}(\omega) \exp \left(i\left[q_{l} n-\omega t\right]\right) d \omega \\
& =\sum_{l}\left\langle q_{l}, \omega \mid f\right\rangle\left|q_{l}, \omega\right\rangle .
\end{aligned}
$$

However, only plane waves that are also invariant under the map Eq. (20) will appear in the equation above. We call them resonant plane waves or plane waves with $\hat{S}$ symmetry. They satisfy $u_{n+r}\left(t+T_{F}\right)=u_{n}(t)$, which implies that $q r-\omega_{L} T_{F}=2 \pi m$, for some integer $m$, and therefore $\omega_{L}=$ $(q r+2 \pi m) / T_{F}$ or

$$
\omega_{L}=q V_{b}+m \omega_{F} ;
$$

that is, the laboratory frequency is the sum of a frequency due to the velocity $q V_{b}$ plus one internal frequency of the MB (an integer multiple of the fundamental frequency).

They are parallel straight lines with slope $V_{b}$ and frequency $m \omega_{F}$ at $q=0$. We call them resonant lines or $m$ lines. They can be seen in Fig. 8(b) together with the 2D DFT of a pterobreather with the same symmetry whose profile is plotted in Fig. 8(a).

Therefore, the plane waves $u_{n}(t)$ can be written as

$$
u_{n}(t)=A \exp \left(i q\left[n-V_{b} t\right]\right) \exp \left(-i m \omega_{F} t\right)
$$

that is, the product of a function with constant shape in the moving frame and a periodic function with a frequency multiple of $\omega_{F}$. However, the combination of both exponential shapes is seen in the laboratory frame as a plane wave traveling with phase velocity $V_{p h}=\omega_{L} / q$. The phase velocity is different from $V_{b}$, different from each $q$, and, if $q$ is negative, it will travel in the opposite direction as the solitary wave with the same symmetry. This becomes apparent in the plane waves that are wings of solitary waves.

Equations (29) have been obtained in Refs. [57,62] in a different form in terms of some breather frequency, which we will define with precision below.

As $V_{b}$ and $\omega_{F}$ are not independent, we can also write

$$
\omega_{L}=\left(r \frac{q}{2 \pi}+m\right) \omega_{F}=\left(q+\frac{2 \pi m}{r}\right) V_{b},
$$

for any $m$ integer. This equation has also been deduced in a more complex form in Ref. [57] [see Eq. (38)] for a $\beta$-FPU system.

Note that there are two complementary options to interpret Eqs. (29)-(31).

\section{A. Representation in the first Brillouin zone (1BZ)}

We can suppose that $q$ is in the first Brillouin zone (1BZ), because for any integer $l$, and other representative $q^{\prime}=q+$ $2 \pi l$, with $l$ an integer, $(q+2 \pi l)=m^{\prime} \omega_{F}$, $=$ with $m^{\prime}=l r+$ $m$ just another integer, which is included in Eq. (29). Also, it is possible to choose the $1 \mathrm{BZ}$ in $[-\pi, \pi]$ or $[0,2 \pi]$, the latter being more convenient for hard potentials as the breather is near $q=\pi$, and in this way it is along a single resonant line. This has been the choice for Fig. 8. (a)

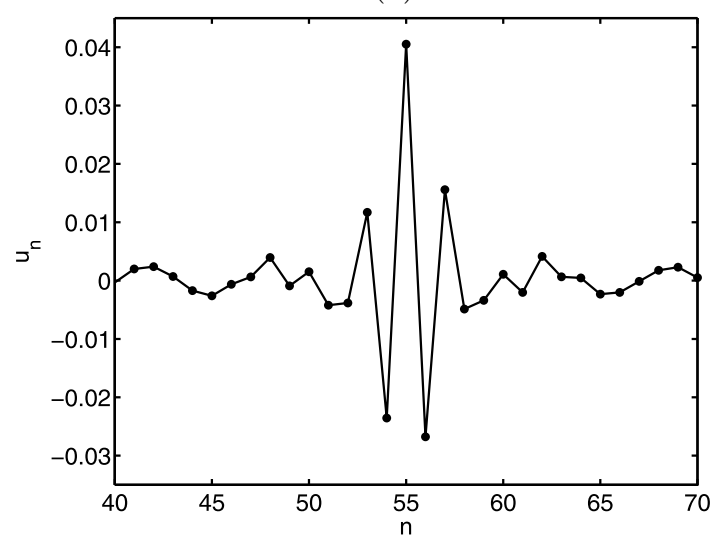

(b)

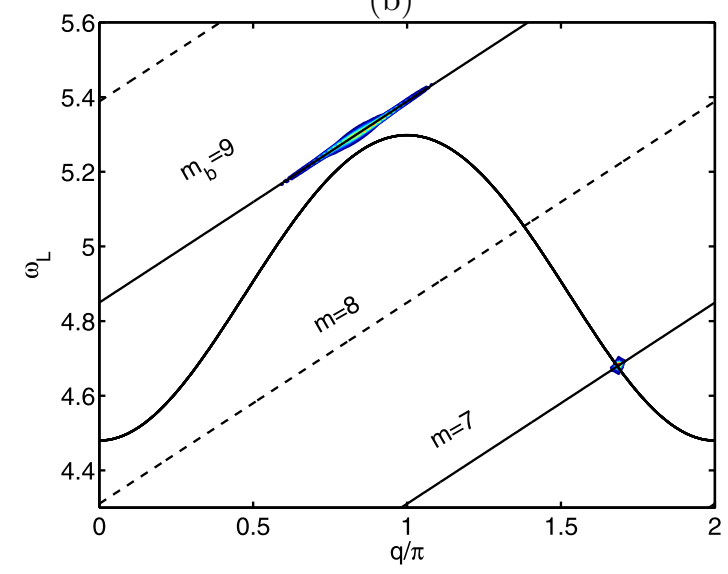

FIG. 8. (a) Pterobreather with fundamental frequency $\omega_{F}=$ $2 \pi / T_{F}=0.53883$ that advances two sites (step $r=2$ ) in $m_{b}=9$ internal oscillations in the fundamental time $T_{F}$. (b) Linear dispersion of the system $\omega_{L}(q)$ in the Brillouin zone $[0,2 \pi]$ and resonant straight lines given by Eq. (29) or (31): $\omega_{L}=q V_{b}+m \omega_{F}$. The line $m_{b}=9$ is the breather line corresponding to the pterobreather. Also plotted are the contour lines of the $2 \mathrm{D}$ fast Fourier transform of the pterobreather. The lines are equivalent modulo $r$ in some extended Brillouin zone. Only the lines $m_{b}=9$ and $m=m_{b}-r=7$ have observable intensity in the 2D DFT (or 9 and 11 in the $[-\pi, \pi]$ representation). The $\pi$ or rest breather frequency $\omega_{b}$ is the frequency in the breather line corresponding to $q=\pi$, and as demonstrated in Sec. VIIIE is $\omega_{b}=s \omega_{F}$ with $s=m_{b}+r / 2$. For this pterobreather $\omega_{b}=5.3883$ and $s=10$. The unit of angular frequency corresponds to $\simeq 0.8 \mathrm{THz}$.

\section{B. Representation in the extended Brillouin zone (EBZ)}

On the other hand if we allow $q$ to be in the extended Brillouin zone, there are only $r$ resonant lines because, using Eq. (31), the resonant line for $m^{\prime}=m+l r$, with any integer $r$ can be written as

$\omega_{L}=V_{b}\left(q+\frac{2 \pi}{r}[m+r l]\right)=V_{b}\left([q+2 \pi l]+\frac{2 \pi}{r} m\right)$.

That is, it is also the resonant line for $m$ in a BZ displaced $l$ $\mathrm{BZs}$ from the original.

Therefore, the indexes $m$ are equivalent modulo $r$. A possible choice of independent $m$ 's are $m=0,1, \ldots, r-1$. The line $m=0$ is a particular one, because $\omega_{L}$ is simply 


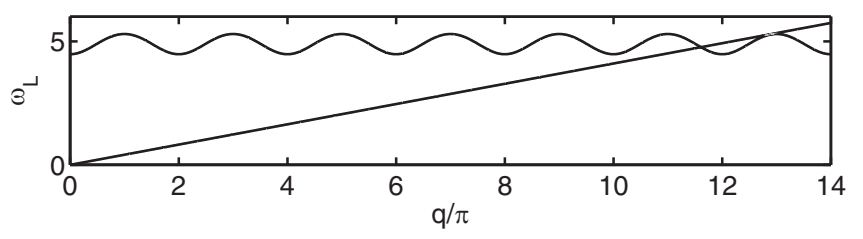

FIG. 9. Plot in the extended Brillouin zone (EBZ) of the 2D DFT (difficult to see), the dispersion relation, and the single resonant line $m=0$, that is, $\omega_{L}=q V_{b}$. This situation is always possible when the step $r=1$. All the phase velocities of the plane waves that form the breather and the wings are in the resonant line and, therefore, have the same velocity and the breather has no internal frequency. It is a rigid profile traveling with velocity $V_{b}$. However, this is done at the cost of a complex structure in $q$ space.

$\omega_{L}=q V_{b}$, i.e., all the plane waves travel at the same velocity $V_{b}$ and the components of a moving solution in this line correspond simply to a rigid profile traveling with speed $V_{b}$. In this representation and case the breather has become a soliton. For any other $m \in\{1, \ldots, r-1\}$, the moving solution has an internal frequency $\omega_{L}=m \omega_{F}=m \omega_{V} / r$, i.e., a fraction $m / r$ of the velocity frequency. For exact solitary waves with $r=1$, the representation in the whole extended zone is always possible.

An example can be seen in Fig. 9. An exact moving breather (with wings) with $m_{b}=6$ and step $r=1$ is represented in the DFT translated to the seventh BZ and a single resonant line with $m=0$. The intensity is in the resonant line close to the quasitangent point with the dispersion relation.

Note, however, that the choice of the extended Brillouin zone simplifies the spectrum of the frequencies but translates this complexity to the wave vector. There is no actual sampling of the spatial variable with values closer than $n$ integer; therefore, the use of the EBZ is somewhat artificial.

\section{The breather line}

Equation (29) determines the value of the resonant $\left(q, \omega_{L}\right)$ plane waves representatives in the first BZ. They determine straight lines, with slope $V_{b}$, that lead to wave packets traveling with velocity $V_{b}$. One of these $m$ lines, say, with $m=m_{b}$ and which we will call the breather line, is of particular interest as the plane waves in this line will have most of the intensity. The breather line in hard potentials is above and close to tangent to the dispersion curve for some value of $q$ near $q=\pi$. In the $[0,2 \pi] \mathrm{BZ}$, there is only one line as $q=\pi$ is in the middle of the interval.

The breather line is below and close to tangent near $q=0$ for soft potentials. In this case the $[-\pi, \pi] \mathrm{BZ}$ can be more convenient.

\section{The wing line(s)}

When a resonant $m$ line cuts the dispersion curve, it will allow for a localized resonant intensity localized in $q$ and $\omega_{L}$. This intensity corresponds to a extended plane wave or a wing. We call them wing lines. There are only a few crossing points in a given Brillouin zone, often only one. Also, most of the intensity is detectable in only one $[57,62]$. The wing line with significant intensity is $m=m_{b}-r$ because it coincides with the breather line in the $[-\pi, \pi]$ representation. In some specific cases the wing line is quasitangent to the dispersion relation producing large wings.

Note that phonons in a nonlinear system have zero amplitude, so they cannot form the wings. The wings are formed by a nonlinear wave with components in the $m$-resonant line in the vicinity of the dispersion relation and above it in a system with hard potential. However, when the amplitude of the wings is very small, it is very close to the phonon in the crossing point.

\section{E. The (rest) or $\pi$-breather frequency}

For hard potentials as it is our interest the breather line will have a frequency $\omega_{b}$ above the phonon band at $q=\pi$. The mode $q=\pi$ does not travel, and thus we can call $\omega_{b}$ the rest breather frequency or the $\pi$-breather frequency. This is the frequency of the stationary breather which can be used to obtain moving breathers with a suitable perturbation. The frequency $\omega_{b}$ can be written as

$$
\omega_{b}=s \omega_{F}, \quad \text { with } \quad s=m_{b}+\frac{r}{2} .
$$

If $r=2$ or even, $s$ is an integer and $\omega_{b}$ is an integer multiple of $\omega_{F}$. If $r=1$ or odd, then $\omega_{b}$ is a half-integer multiple of $\omega_{F}$. The latter case may seem strange, but the actual breather frequency is the one in the moving frame as we will see below. Indeed, for $r$ odd, $s$ is always a half-integer.

For soft potentials the (rest) breather frequency will be the frequency of the mode $q=0$ in the breather line and $s=m_{b}$.

\section{F. The breather internal frequency in the moving frame}

In terms or the (rest) breather frequency $\omega_{b}$ the breather line can be written as

$$
\omega_{L}=\omega_{b}+V_{b}(q-\pi)
$$

and the corresponding plane waves

$$
u_{n}(t)=\exp \left(i q\left[n-V_{b} t\right]\right) \exp \left(-i\left[\omega_{b}-\pi V_{b}\right] t\right) .
$$

As the second exponential does not depend on $q$, all the plane waves in the breather line have the same internal frequency

$$
\omega_{i, b}=\omega_{b}-\pi V_{b}=m_{b} \omega_{F},
$$

which will be the main internal or traveling frequency of the moving breather.

\section{G. The wing internal frequencies in the moving frame}

As we have seen above there are other frequencies when the $m$ lines cross the dispersion relations. They have to be inside the $m$ line, not very far from the linear dispersion curve and above it for a hard on-site potential. The internal frequencies in the moving frame are thus $m \omega_{F}$ for the $m$ lines crossing the dispersion relation. There are very few, often only one. Even when there are more than one, only one has a significant intensity and therefore only a wing frequency with $m=m_{b}-r$.

This can be applied, for example, to a pterobreather with $r=2$ and $m_{b}=9$. There are two values of the $\omega_{L}$ and $m$ 


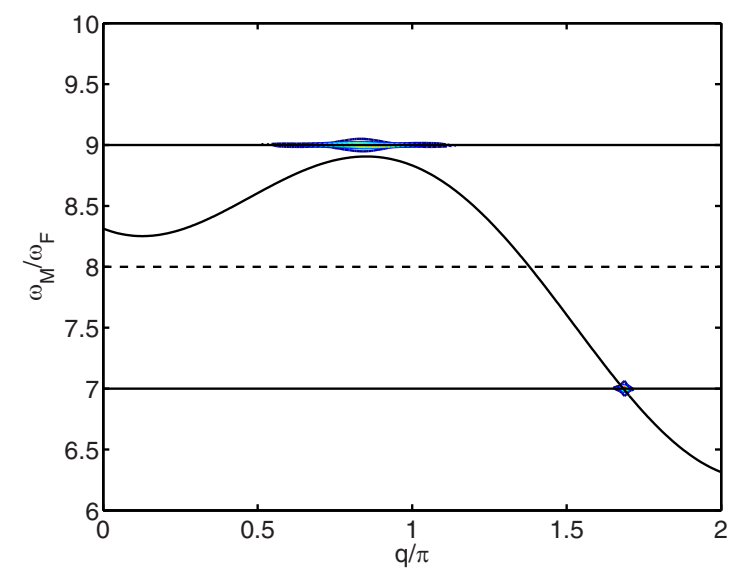

FIG. 10. Frequencies $\omega_{M}$ in the moving frame that travels with the solitary wave relative to the fundamental frequency $\omega_{F}$. The 2D DFT corresponds to the same pterobreather with $r=2, m_{b}=9$, and $s=m_{b}+r / 2=10$ represented in Fig. 8. The curved line is the dispersion relation (DR) in the moving frame. The possible internal frequencies are $\omega_{m}=m \omega_{F}$, with $m$ integer, and correspond to horizontal lines with integer relative frequency. As the plane waves with some intensity have to be relatively close to the DR and for hard potentials above it, the breather line is the next integer horizontal line above the DR. Crossing points of $m$ lines with the DR give extended wings. There are two possible crossing points, but only the one with $m=7$ has measurable intensity.

solution of Eq. (29) and (13), as can be seen in Fig. 8. They correspond to $m=7$ and 8 . The breather line corresponds to $m_{b}=9$ and the wing line to $m=7$.

\section{H. The $\omega-q$ representation in the moving frame}

The picture is much clearer if the frequencies are measured in the moving frame and with values relative to the fundamental frequency as seen in Fig. 10. The possible internal frequencies are $\omega_{m}=m \omega_{F}$ and correspond to horizontal lines with integer relative frequency. In the figure, the curved line is the dispersion relation (DR) in the moving frame. For a given line to have intensity, it has to be relatively close to the dispersion relation and for hard potentials above it. For a localized wave packet there is the need of an interval in $q$; therefore, the breather line is the closest integer horizontal line above the maximum of the DR, where it will have the maximum intensity. The corresponding $q$ will be the main wave number for the breather.

Frequencies near the crossings point of the DR with integer horizontal lines produce extended waves of wings. In the figure there are two possible crossing points, but only one of them with $m=7$ has measurable intensity.

The reason for this is more apparent in the $\omega$ - $q$ representation of the same pterobreather in the laboratory frame in Fig. 8. The $m=7$ line is the prolongation of the $m_{b}=9$ line into the $[-\pi, 0]$ interval; therefore, it is also the breather line. In the moving frame representation of Fig. 10 can also be seen that the breather line, with $m=9$, will cut the DR when both are continued into the $[-\pi, 0]$ interval. The mathematical equivalence of the resonant $m$ and $m \pm r$ lines has been demonstrated in Sec. VIII B.
However, it is possible that there is some intensity near the crossing point of the line $m=8$ with the DR, although we could not detect it. In other systems, the intensity for other resonant frequencies is some orders of magnitude smaller than the one with more intensity [57,62].

For nonexact solitary waves, the horizontal lines can have noninteger values.

\section{STABILITY OF EXACT MOVING BREATHERS}

Exact moving solutions are a function solution of the dynamical equations

$$
\ddot{u}-F(u)=\ddot{u}+\frac{\partial H}{\partial u}=0,
$$

which is invariant under time translations $t \rightarrow t+t^{\prime}, \forall t^{\prime}$ as the system is autonomous and by the translations $n \rightarrow n+d$ for any $d$ integer. In practice lattices are finite with dimension $N$ and $d<N$.

Exact moving solutions are fixed points of the map in Eq. (20):

$$
\hat{S}:\left\{u_{n}(t)\right\} \rightarrow\left\{u_{n+r}\left(t+T_{F}\right)\right\},
$$

for some integers $r$ and time $T_{F}$.

Let us call $u^{0}$ a solution with the symmetry above, and $\xi(t)$ a perturbation of it with momenta $\pi(t)=\dot{\xi}(t)$. Then $u^{0}+\xi$ is also a solution of Eq. (37). If we suppose that $\xi$ is small, Eq. (37) can be linearized with respect to $\xi$ and $\pi$ and belongs to the kernel of the Newton operator $\mathcal{N}$ defined as

$$
\mathcal{N} \equiv \dot{\pi}-F^{\prime}\left(u^{0}\right) \xi
$$

that is,

$$
\mathcal{N} \xi=0
$$

The operator $\mathcal{N}$ has the symmetries of the solution $u^{0}$ as defined in Eq. (20). A solution $\xi(t)$ is defined by its initial (small) values $\xi_{n}(0)$ and $\pi_{n}(0)$. After integrating Eq. (39) at time $T_{F}$, the values of $\xi_{n}\left(T_{F}\right), \pi_{n}\left(T_{F}\right)$ and $\xi_{n+r}\left(T_{F}\right), \pi_{n+r}\left(T_{F}\right)$ can be obtained. In this way the modified Floquet matrix $\mathcal{F}_{r}$ can be defined:

$$
\left[\begin{array}{c}
\left\{\xi_{n+r}\left(T_{F}\right)\right\} \\
\left\{\pi_{n+r}\left(T_{F}\right)\right\}
\end{array}\right]=\mathcal{F}_{r}\left[\begin{array}{l}
\left\{\xi_{n}(0)\right\} \\
\left\{\pi_{n}(0)\right\}
\end{array}\right] .
$$

Due to the symplectic nature of the system the eigenvalues corresponding to a stable solution have to be in the unit circle. Integration of the solution with $\left(r=1, m_{b}=6\right)$ leads to the eigenvalues represented in Fig. 11. For moving breathers there are four eigenvalues in $(1+0 i)$ related to the proximity of other solutions with a shift in frequency or localization, velocity, and the two initial phases in each variable in Eq. (23) [63]. These phases correspond to the change in initial time (phase mode) and initial position. Therefore, four eigenvalues at $1+0 i$ do not imply structural instability as they cannot leave that position. This is different from stationary breathers where they would imply structural instability. In this way the stability of the pterobreathers is calculated, and they are mostly stable as in the example shown. 


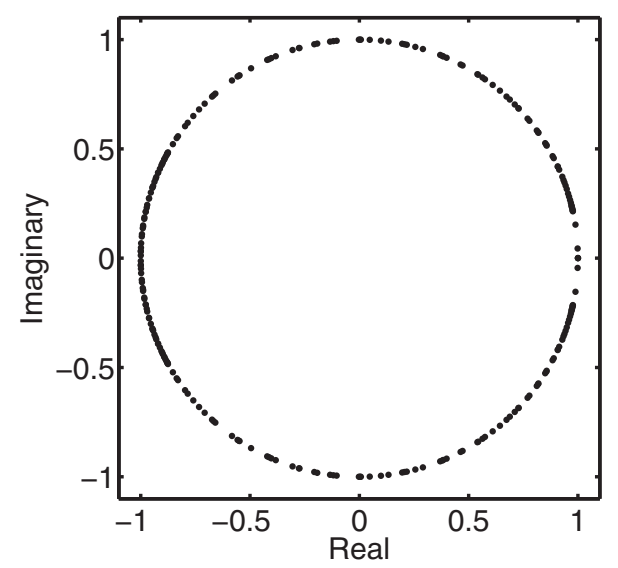

FIG. 11. Eigenvalues of the modified Floquet $\mathcal{F}_{r}$ matrix for a pterobreather with $r=1$ and $m_{b}=6$, which is stable because all the eigenvalues are in the unit circle. There are four eigenvalues in $1+0 i$, although it cannot be appreciated in the plot. This is a typical feature of moving breathers [63], which does not imply structural instability.

\section{EXAMPLES OF EXACT PTEROBREATHERS WITH SMALL WINGS}

We have been able to obtain a few thousands of stable pterobreathers with small wings and different symmetries parameterized by the step $r$ and the parameter $m_{b}$, i.e., the number of internal oscillations while the breather moves $r$ sites and repeats its profile. The other parameter should be

TABLE I. Examples of pterobreathers and exact moving breathers obtained. Key: $r$, step; $m_{b}$, number of internal oscillations to advance $r$ sites; $\omega_{b}=s \omega_{F}$, frequency of the mode $\pi$ in the breather line with $s=m_{b}+r / 2 ; V_{b}$, velocity; $E_{b}$, core breather energy in scaled units (sc) or eV; $N_{\text {eff }}$, effective number of particles. The core energy is obtained as the sum of local energies $N_{\text {eff }}$ at both sides of the particle with maximum amplitude. Scaled units of velocity and frequency are $\simeq 2.6 \mathrm{~km} / \mathrm{s}$ and $0.8 \mathrm{THz}$.

\begin{tabular}{ccccccc}
\hline \hline$\omega_{b}$ & $r$ & $m_{b}$ & $V_{b}$ & $E_{b}(\mathrm{sc})$ & $E_{b}(\mathrm{eV})$ & $N_{\text {eff }}$ \\
\hline \multicolumn{5}{c}{ Pterobreathers } \\
\hline 5.35 & 1 & 5 & 0.155 & 0.043 & 0.12 & 12 \\
5.35 & 1 & 6 & 0.131 & 0.053 & 0.15 & 9 \\
5.35 & 1 & 7 & 0.114 & 0.059 & 0.16 & 8 \\
5.35 & 1 & 8 & 0.100 & 0.063 & 0.17 & 8 \\
5.35 & 2 & 9 & 0.170 & 0.034 & 0.09 & 15 \\
5.35 & 2 & 10 & 0.155 & 0.043 & 0.12 & 12 \\
5.39 & 2 & 11 & 0.143 & 0.082 & 0.23 & 8 \\
5.35 & 2 & 12 & 0.131 & 0.053 & 0.15 & 9 \\
5.35 & 2 & 13 & 0.122 & 0.057 & 0.16 & 9 \\
5.35 & 2 & 14 & 0.114 & 0.059 & 0.16 & 8 \\
5.35 & 2 & 15 & 0.106 & 0.061 & 0.17 & 8 \\
5.35 & 2 & 16 & 0.100 & 0.063 & 0.17 & 8 \\
& & & Exact moving breathers & & \\
\hline 5.34 & 1 & 6 & 0.131 & 0.037 & 0.10 & 13 \\
5.33 & 1 & 7 & 0.113 & 0.039 & 0.11 & 13 \\
5.35 & 2 & 9 & 0.170 & 0.034 & 0.09 & 15 \\
\hline \hline
\end{tabular}

$\omega_{F}$ or $\omega_{b, i}$, but instead we use the (rest) breather frequency $\omega_{b}=s \omega_{F}$. This is because $\omega_{b}$ has a more intuitive meaning in the laboratory frame as the frequency of the related stationary breather and as the frequency where the breather line cuts the vertical line $q=\pi$ in the $\omega$ - $q$ diagrams, which is easy to visualize. However, it should be remembered that the moving breather has a single frequency $\omega_{i, b}$ only in the moving frame.

We have obtained pterobreathers for many symmetries as $r=1, m_{b}=5, \ldots, 8$ and $r=2, m_{b}=9, \ldots, 16$, with $\omega_{b}$ in a small range of approximately 5.3-5.4, although the exact range depends on the particular symmetry. Larger frequency leads to very large wings or complete delocalized solutions, and smaller frequencies lead to delocalized quasiphonons. Some examples are presented in Table I. They have wings that are of the order of $10^{-6}$ while the breather amplitudes are of the order of $10^{-2}$. Below we give some details about a specific one, but the differences are not significant from one to other.

(a)

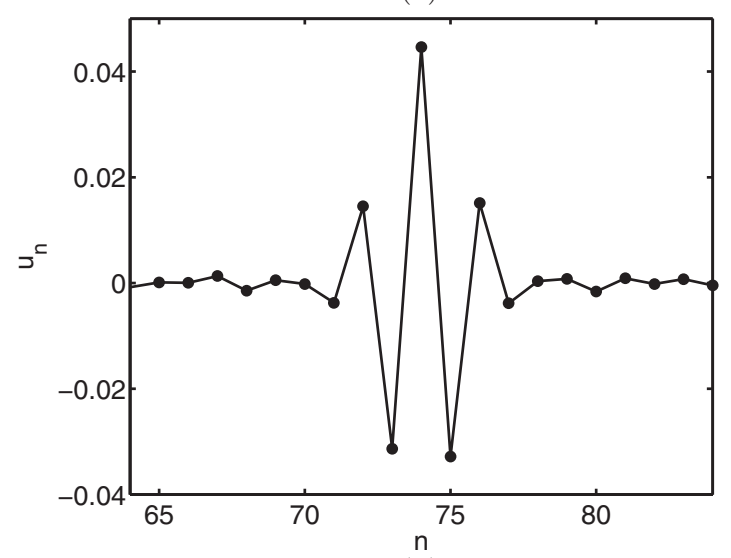

(b)

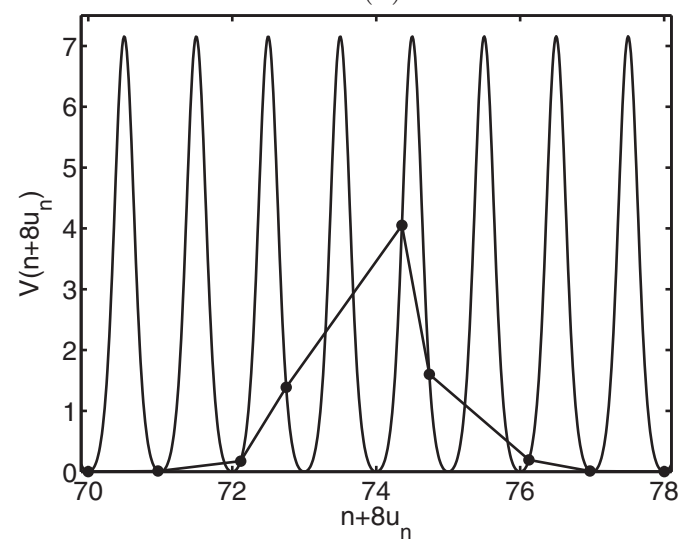

FIG. 12. (a) Profile $\left(n, u_{n}\right)$ of a pterobreather. (b) For the same pterobreather, representation $\left[n+8 u_{n}, V\left(n+8 u_{n}\right)\right]$, where $V$ is the substrate potential. The displacements have been amplified eight times in order that the pterobreather structure can be easily appreciated. Both profiles correspond to the situation where a particle $n_{0}=74$ has the largest amplitude, and it is nearly at rest. Note the asymmetry with respect to the central particle because the repulsion between the $n_{0}+1$ particle is much larger than with the $n_{0}-1$ one. The particle $n_{0}-1$ is moving to the left and $n_{0}+1$ is moving to the right with similar velocities. Data: Step $r=2, m_{b}=11, \omega_{b}=$ 5.3933. The units of distance is the lattice unit $5.19 \AA$ and of energy $\simeq 2.77 \mathrm{eV}$. 


\section{A. Example of exact pterobreather with $m_{b}=11$ and $r=2$}

An example is an exact pterobreather that advances $r=2$ sites while performing $m_{b}=11$ internal oscillations, that is, with $s=m_{b}+r / 2=12$. Its frequency is $\omega_{b}=5.3933$ and localization $N_{\text {eff }} \simeq 8$. Its amplitude $A=0.045$ with wings $10^{-2}$ times smaller. Its velocity is $V_{b}=0.1415$. The core energy is $0.2277 \mathrm{eV}$, and it is much more localized than the amplitude, being the energy per site of the wings $10^{-5}$ smaller than the central site. The profiles $\left(n, u_{n}\right)$ and amplified ones $\left(n+u_{n}, V_{n}\right)$ are shown in Fig. 12 for the configuration in which a particle $\left(n_{0}\right)$ has maximum amplitude. Note the asymmetry with respect to the two neighbors due to being closer to the next particle ahead than to the one behind.

Exact pterobreathers will not exist in a real system, but a good approximation of them will be able to carry energy along the system. Moreover, there exist also some values of frequencies and symmetries for which there are exact moving breathers with wings, as is studied in the next section.

\section{EXAMPLES OF EXACT MOVING BREATHERS WITHOUT WINGS}

By path continuation from an exact pterobreather, keeping constant the step $r$ and $m_{b}$ and changing the $\pi$-breather frequency $\omega_{b}$, it is possible to find exact moving breathers without wings. Figure 13 shows the amplitudes of the wings $A_{w}$ as a function $\omega_{b}$ and how it becomes zero within the precision of the calculation. Also, the shape of the curves $A_{w}\left(\omega_{b}\right)$ suggests that they tend exactly to zero. Exact moving breathers are also characterized by the exponential decay of the amplitudes as can be seen in Fig. 14(b).

For example, by continuation of a pterobreather with parameters $r=1, m_{b}=6$, and $\omega_{b}=5.35$, the amplitude $A\left(\omega_{b}\right)$ of the wings diminishes with diminishing frequency, and the wings disappear for $\omega_{b}=5.3353$ and $V_{b}=0.13063$. The amplitude of each particle can be obtained as the maximum

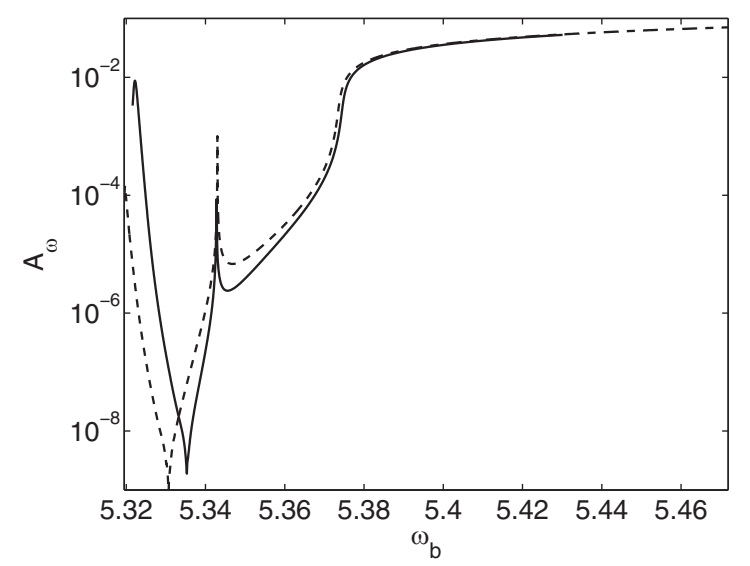

FIG. 13. Logarithmic plot of the wing amplitudes as a function of the rest breather frequency for two cases with step $r=1$ and $m_{b}=6$ (continuous line) or 7 (dashed line). It can be seen that the wings disappear within numerical precision for specific frequencies. Similar curves have been obtained with parameters $r=2$ and $m_{b}=9, \ldots, 16$ with slightly different frequencies when the wing amplitude tends to zero. Units are lattice units $u_{L}=5.19 \AA$. (a)

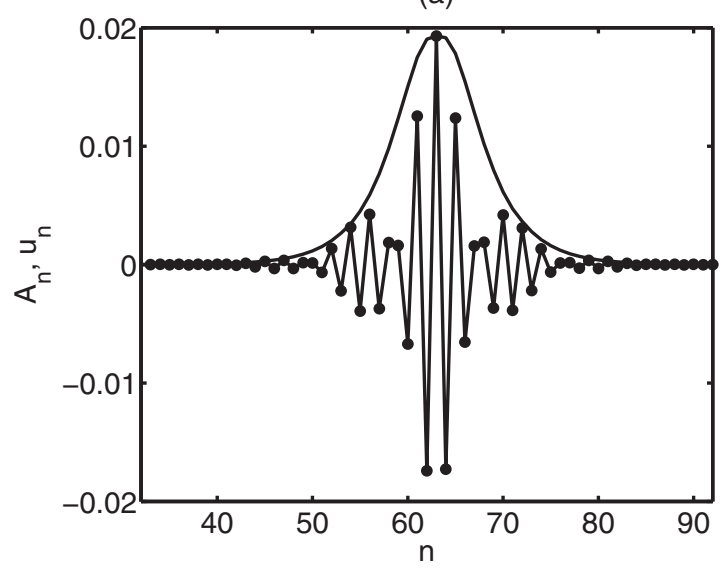

(b)

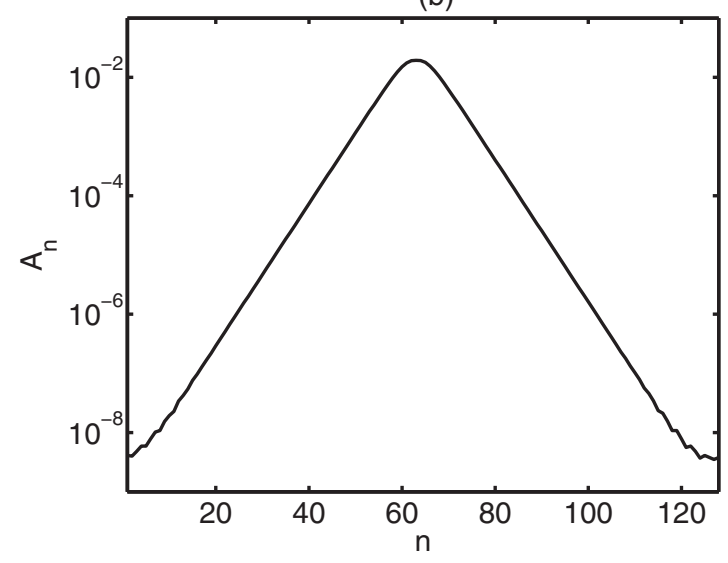

FIG. 14. (a) Amplitudes and displacements of the exact breather solution with step $r=1$ and $T_{F}=T_{b} / 6.5$ obtained at frequency $\omega_{b}=$ 5.3353 and $V_{b}=0.13064$. The amplitudes have an exact exponential decay $\exp \left(-\xi\left|n-n_{0}\right|\right)$ a couple of particles far from the core, with $\xi=0.278$ as can be seen below. (b) Logarithmic plot of the amplitudes of the exact breather where the exponential decay can be seen. The unit of distance is equal to the lattice unit $u_{L}=5.19 \AA$.

absolute value of its position during an interval of time $T_{F}$, i.e., $A_{n}=\max \left|u_{n}(t)\right|$, for $t \in\left[0, T_{F}\right)$. It has a perfect exponential decay with $n$ and $\xi=0.278$ as can be seen in Fig. 14(b), with an effective number of particles $N_{\text {eff }} \simeq 18$. The amplitudes $A_{n}$ fit very well to the function $\operatorname{sech}[\xi(n-$ $\left.n_{0}\right)$ ], which approaches $2 \exp \left(-\xi\left|n-n_{0}\right|\right)$ a few units far from $n_{0}$.

Its energy is $E=0.037$ in scaled units or $0.103 \mathrm{eV}$, which is compatible with the estimated energy for secondary quodons in fossil tracks of muscovite.

The potential and kinetic energy oscillate in opposition of phase around $E / 2$, with a frequency $2 \omega_{b}=13 \omega_{F}$, that is, doing 13 oscillations in the time for advancing one site. They can be written approximately as $E_{k}=E[1 / 2-$ $\left.0.07 \cos \left(2 \omega_{b} t\right)\right]$ and $E_{\text {pot }}=E\left[1 / 2+0.07 \cos \left(2 \omega_{b} t\right)\right]$. This is coherent with the displacement and velocities being approximately proportional to $\cos \left(\omega_{b} t\right)$ and $\sin \left(\omega_{b} t\right)$ and the potential and kinetic energies being proportional to the corresponding second power. That is, the energies can also be written $E_{k}=E\left[0.43+0.14 \sin ^{2}\left(\omega_{b} t\right)\right]$ and $E_{\text {pot }}=E[0.43+$ $\left.0.14 \cos ^{2}\left(\omega_{b} t\right)\right]$, the latter can also be decomposed in substrate 
(a)

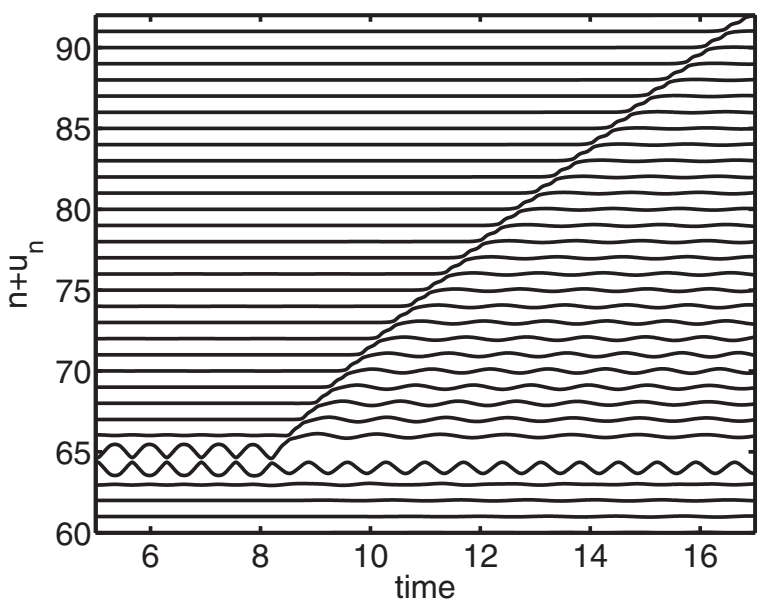

(b)

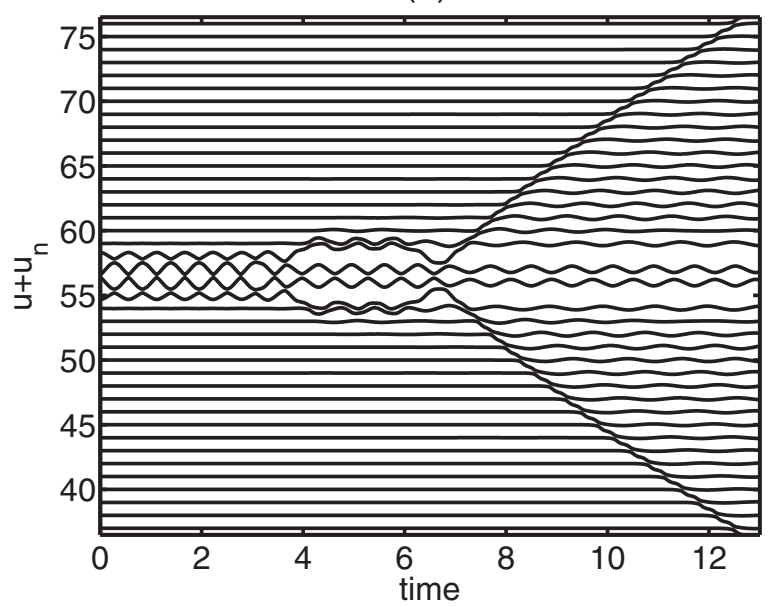

(c)

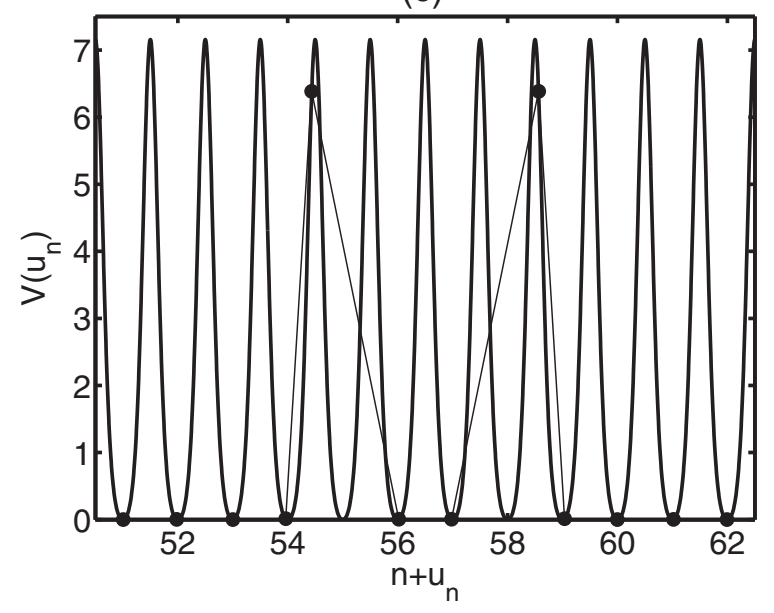

FIG. 15. High-energy breathers producing kinks. (a) An unstable breather experiments a symmetry breaking. See text for details. (b) A breather of large energy that is unstable and after several oscillations produces a couple of kinks traveling in opposite directions. (c) Plot of $\left[n+u_{n}, V\left(u_{n}\right)\right]$, where $V$ is the substrate potential, of the system after the kinks have just been formed, and two particles moving outwards have left behind two vacancies. The two particles in the center will oscillate with large amplitude and symmetry with respect to their bond. Scaled units: for $n$ and $u_{n}$ the lattice spacing $a=5.19 \AA$, and the unit of energy is $2.77 \mathrm{eV}$. potential energy $E_{\text {sub }}=E\left[0.31+0.1 \cos ^{2}\left(\omega_{b} t\right)\right]$ and interaction potential energy $E_{\text {int }}=E\left[0.12+0.04 \cos ^{2}\left(\omega_{b} t\right)\right]$. In this last point there is a baseline energy of $0.86 E$ upon which the kinetic and potential energies are added.

The exact breather is stable with all the Floquet eigenvalues as defined in Eq. (40) with modulus unity and four eigenvalues at the $1+0 i$ as explained in Sec. IX.

Minimal changes occur for the breather without wings and symmetry $r=1, m_{b}=7$, the energy being slightly larger, $E \simeq 0.108 \mathrm{eV}$, but the frequency and velocity slightly smaller, $\omega_{b}=5.3307$ and $V_{b}=0.11312$, both about one-third of the maximum group velocity, i.e., the sound velocity $0.409 \simeq$ $1 \mathrm{~km} / \mathrm{s}$.

As there are many other symmetries, there are many other exact breathers without wings, being a discrete set among the pterobreathers, in the same way that there is a finite set of kinks without wings [24]. They provide a set of supertransmission channels for low energies without mass transfer.

\section{HIGH-ENERGY STATIONARY BREATHERS}

There are some interesting examples of stationary breathers with high energy and frequency, which we describe in this section.

\section{A. Stationary breathers that produce kinks}

For large energies a bond-symmetric, stationary breather, which is unstable, can transform into a single kink or crowdion with a symmetry breaking phenomenon. The kink usually has wings as the energy delivered is larger than the sliding kink that exists in the system $[23,24]$. It leaves a vacancy and a vacancy-nonlinear localized mode behind, as can be seen in Fig. 15(a). It can be observed that the kink slows down because it is not an exact solitary wave. Their velocity will tend asymptotically to the sliding kink solution. Also, in a periodic system when the kink interacts again with the vacancy, it rebounds.

For larger energies, the bifurcation can be into a couple of kinks or crowdions with wings that leave two vacancies separated by two particles behind vibrating with high energy. In this case, the symmetry is preserved. This phenomenon can be seen in Figs. 15(b) and 15(c). The breather energy is $69.4 \mathrm{eV}$, enough for producing two $27 \mathrm{eV}$ sliding kinks and leaving substantial vibrational energy behind.

\section{B. Extremely localized multibreathers}

At high frequencies, there are extremely localized multibreathers for frequencies of the order of $\omega_{b} \simeq 9-10$. Breathers and multibreathers exist being site-centered for an odd number of excited particles and bond-centered for an even one. They are extremely localized at those frequencies, but some of the site-centered ones are stable.

One example consists of three excited particles with frequency $\omega_{b}=9.19$ and energy $69.4 \mathrm{eV}$. The situation of maximum displacement for the central particle is represented in Fig. 16. The velocities are zero, and the neighboring particles are at the maximum displacement in their direction. 


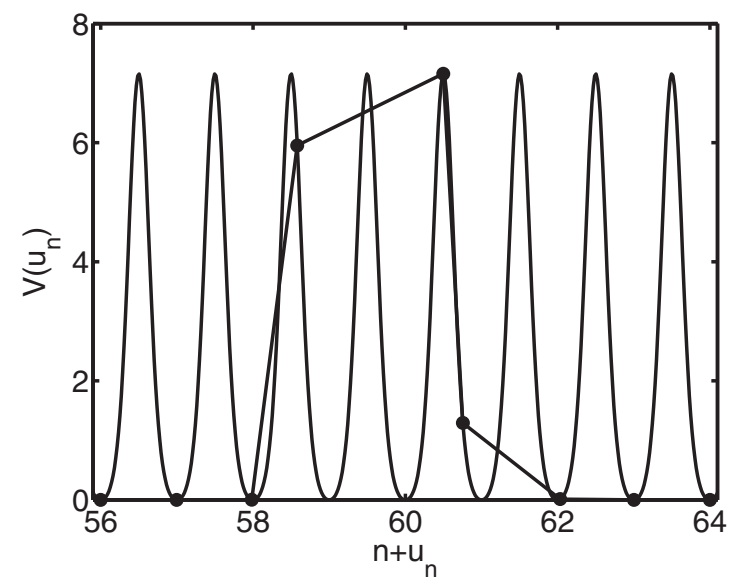

FIG. 16. Plot of $\left[n+u_{n}, V\left(u_{n}\right)\right]$, where $V$ is the substrate potential, of an extremely localized, stable, three-site breather at the position where all the velocities are zero and the particles are at the maximum displacement in their direction. After half a period the situation will be reversed, i.e., $u_{n}\left(t+T_{b} / 2\right)=-u_{n}(t)$. Frequency $\omega_{b}=9.19$. Scaled units for energy, equivalent to $2.77 \mathrm{eV}$. For distances, the lattice unit $5.19 \AA$.

Note that there is no symmetry with respect to the central particle at that instant; the symmetry is given by $u_{n}(t+$ $\left.T_{b} / 2\right)=-u_{n}(t)$. The neighboring particles have smaller amplitudes than the central one.

At this frequency, the central particle at the initial time is almost at the top of the potential barrier, but it is also possible to observe the situation when the maximum displacement is at the other potential well, thereafter being repelled by the neighboring particle and coming back to its own well. The neighbors have amplitudes $[0.84,0.07,0.005, \ldots]$ diminishing exponentially from the second neighbor.

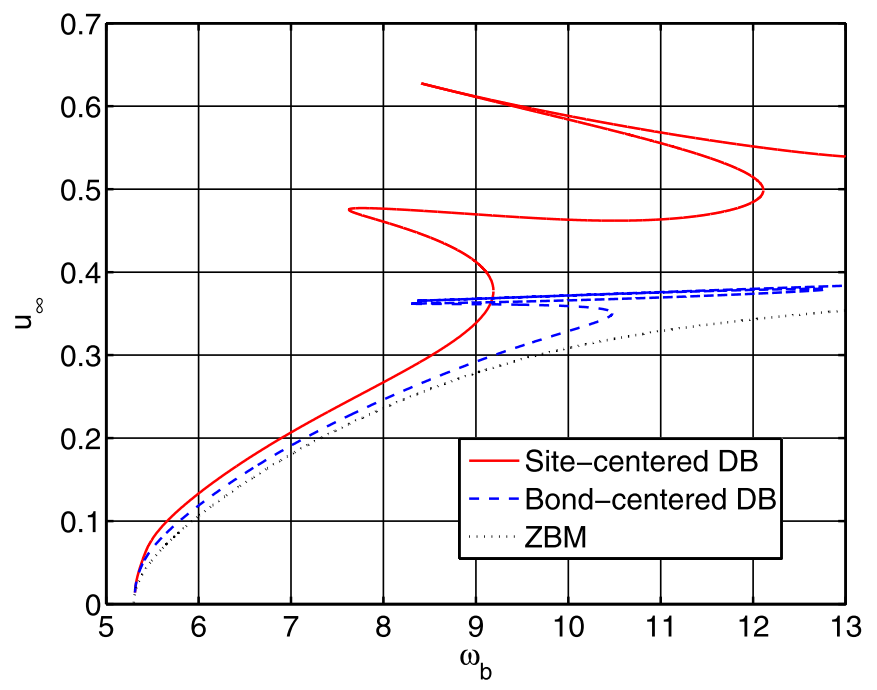

FIG. 17. Bifurcation diagram of the maximum amplitude as a function of the frequency of stationary breathers. For several frequencies there coexist different types of breathers. ZBM means zone boundary mode, i.e., the nonlinear continuation of the extended mode from which the breathers are derived. For hard potentials, as in our system, this is the $\pi$ mode. The ordinate units are the lattice unit.

\section{Bifurcation diagram}

By path continuation it is possible to obtain the bifurcation diagram as a function of different parameters. These diagrams are quite complex and often involve multibreathers. The bifurcation diagram of the maximum amplitude as a function of the frequency is represented in Fig. 17.

\section{CONCLUSIONS}

In this work we have studied the existence of stationary and moving breathers in a 1D model for the movement of potassium ions in the rows of the cation layer of the silicate muscovite mica. This study is motivated by the fossil and experimental evidence of moving excitations in the cation layer of the mineral with different energies. In previous works was found the existence of kinks with wings and, in particular, a stable single kink without wings. The large energy of the latter makes it a good candidate for primary tracks, but its energy is too large for secondary tracks.

We have found that there exist exact generically moving breathers with wings of small amplitude or pterobreathers. Their energies are of the order of $0.1-0.2 \mathrm{eV}$, which is expected for secondary tracks. The wings are a typical feature of moving breathers in systems with a substrate, but we have also found that for many symmetries there exist exact moving breathers without wings with specific frequencies and energies. They provide a set of supertransmission channels for energy transfer without mass and make them good candidates for some of the secondary tracks.

Motivated by the need for understanding and describing with precision the solutions found, we have also developed a description of exact moving breathers by defining with precision several concepts in the moving frame. In particular the moving breathers have a single frequency and the wings, very few, typically only one. They are integer multiples of a frequency named the fundamental frequency. An extension of these concepts and their application to other potentials, systems. and solitary waves is in preparation and will be published separately.

We have obtained mathematical approximations for the wings and tails of the moving breathers which show very good agreement with the numerical calculations.

We have also studied the properties and energies of lowand high-energy stationary breathers. For the latter we have constructed bifurcation diagrams and observed several interesting phenomena of potential physical interest, as extremely localized multibreathers or the production of kinks by highenergy breathers.

\section{ACKNOWLEDGMENTS}

J.F.R.A. wishes to acknowledge a mobility grant PRX18/00360 from MICINN, project FIS2015-65998C2-2-P from MINECO, and grant 2017/FQM-280 from Junta de Andalucía, Spain. He also gratefully acknowledges Prof. Yusuke Doi and the University of Osaka for hospitality. Y.D. acknowledges the support of Grant-in-Aid for Scientific Research (C) No. 16K05041 from the Japan Society for the Promotion of Science (JSPS). M.K. acknowledges 
support from Kyoto University Foundation and CASIO Science Promotion Foundation (No. H29-17). All authors acknowledge Prof. Kazuyuki Yoshimura, from Tottori
University, Prof. Yosuke Watanabe, from Osaka University, and Prof. Masayuki Sato, from Kanazawa University, for useful discussions.
[1] E. C. H. Silk and R. S. Barnes, Examination of fission fragment tracks with an electron microscope, Philos. Mag. 4, 970 (1959).

[2] P. B. Price and R. M. Walker, Observation of fossil particle tracks in natural micas, Nature (London) 196, 732 (1962).

[3] S. A. Durrani, Nuclear tracks: A success story of the 20th century, Rad. Meas. 34, 5 (2001).

[4] S. A. Durrani, Nuclear tracks today: Strengths, weaknesses, challenges, Rad. Meas. 43, S26 (2008).

[5] F. M. Russell, The observation in mica of tracks of charged particles from neutrino interactions, Phys. Lett. B 25, 298 (1967).

[6] F. M. Russell, Tracks in mica caused by electron showers, Nature (London) 216, 907 (1967).

[7] F. M. Russell, Duration of sensitive period for track recording in mica, Nature (London) 217, 51 (1967).

[8] F. M. Russell, Channelled lepton tracks and practical perspectives, Nucl. Tracks. Rad. Meas. 19, 955 (1991).

[9] F. M. Russell, Decorated track recording mechanisms in muscovite mica, Nucl. Tracks. Rad. Meas. 19, 109 (1991).

[10] F. M. Russell and D. R. Collins, Lattice-solitons in radiation damage, Nucl. Instrum. Meth. B 105, 30 (1995).

[11] F. M. Russell and D. R. Collins, Lattice-solitons and non-linear phenomena in track formation, Rad. Meas. 25, 67 (1995).

[12] F. M. Russell and J. C. Eilbeck, Evidence for moving breathers in a layered crystal insulator at $300 \mathrm{~K}$, EPL 78, 10004 (2007).

[13] X. Mougeot and R. G. Helmer, LNE-LNHB/CEA-Table de Radionucléides, K-40 tables, http://www.nucleide.org (2012).

[14] J. A. Cameron and B. Singh, Nuclear data sheets for $A=40$, Nucl. Data Sheets 102, 293 (2004).

[15] J. F. R. Archilla, Yuriy A. Kosevich, N. Jiménez, V. J. SánchezMorcillo, and L. M. García-Raffi, A supersonic crowdion in mica, in Quodons in Mica: Nonlinear Localized Travelling Excitations in Crystals, Springer Series in Materials Science, Vol. 221, edited by J. F. R. Archilla et al. (Springer, Cham, 2015), pp. 69-96.

[16] J. F. R. Archilla and F. M. Russell, On the charge of quodons, Lett. Mater. 6, 3 (2016).

[17] F. M. Russell, Positive charge transport in layered crystalline solids, Phys. Lett. A 130, 489 (1988).

[18] F. M. Russell, Tracks in mica, 50 years later, in Quodons in Mica: Nonlinear Localized Travelling Excitations in Crystals, Springer Series in Materials Science, Vol. 221, edited by J. F. R. Archilla et al. (Springer, Cham, 2015), pp. 3-33.

[19] F. M. Russell, J. F. R. Archilla, F. Frutos, and S. MedinaCarrasco, Infinite charge mobility in muscovite at $300 \mathrm{~K}$, EPL 120, 46001 (2017).

[20] F. M. Russell, M. W. Russell, and J. F. R. Archilla, Hyperconductivity in fluorphlogopite at $300 \mathrm{~K}$ and $1.1 \mathrm{~T}$, EPL 127, 16001 (2019).

[21] J. F. R. Archilla, Yu A Kosevich, N. Jiménez, V. SánchezGordillo, and L. M. García-Raffi, Moving excitations in cation lattices, Ukr. J. Phys. 58, 646 (2013).
[22] J. F. R. Archilla, Yu. A. Kosevich, N. Jiménez, V. J. SánchezMorcillo, and L. M. García-Raffi, Supersonic kinks in Coulomb lattices, in Localized Excitations in Nonlinear Complex Systems, edited by R. Carretero-González et al. (Springer, Cham, 2014), pp. 317-331.

[23] J. F. R. Archilla, Yu. A. Kosevich, N. Jiménez, V. J. SánchezMorcillo, and L. M. García-Raffi, Ultradiscrete kinks with supersonic speed in a layered crystal with realistic potentials, Phys. Rev. E 91, 022912 (2015).

[24] J. F. R. Archilla, Y. Zolotaryuk, Yu. A. Kosevich, and Y. Doi, Nonlinear waves in a model for silicate layers, Chaos 28, 083119 (2018).

[25] S. Flach and A. V. Gorbach, Discrete breathers: Advances in theory and applications, Phys. Rep. 467, 1 (2008).

[26] M. Remoissenet, Waves Called Solitons: Concepts and Experiments (Springer, Berlin, Heidelberg, 1999).

[27] D. Saadatmand, D. Xiong, V. A. Kuzkin, A. M. Krivtsov, A. V. Savin, and S. V. Dmitriev, Discrete breathers assist energy transfer to ac-driven nonlinear chains, Phys. Rev. E 97, 022217 (2018).

[28] F. M. Russell, Transport properties of quodons in muscovite and prediction of hyper-conductivity, in Nonlinear Systems, Vol. 2: Nonlinear Phenomena in Biology, Optics and Condensed Matter, edited by J. F. R. Archilla et al. (Springer, Cham, 2018), pp. 241-260.

[29] Q. Dou, J. Cuevas, J. C. Eilbeck, and F. M. Russell, Breathers and kinks in a simulated crystal experiment, Discret. Contin. Dyn. Syst. S 4, 1107 (2011).

[30] J. L. Marín, J. C. Eilbeck, and F. M. Russell, Localized moving breathers in a 2D hexagonal lattice, Phys. Lett. A 248, 225 (1998).

[31] J. Bajars, J. C. Eilbeck, and B. Leimkuhler, Numerical simulations of nonlinear modes in mica: Past, present and future, in Quodons in Mica: Nonlinear Localized Travelling Excitations in Crystals, Springer Series in Materials Science, Vol. 221, edited by J. F. R. Archilla et al. (Springer, Cham, 2015), pp. 35-67.

[32] J. Bajars, J. C. Eilbeck, and B. Leimkuhler, Nonlinear propagating localized modes in a 2D hexagonal crystal lattice, Physica D (Amsterdam) 301-302, 8 (2015).

[33] M. Sato, T. Nakaguchi, T. Ishikawa, S. Shige, Y. Soga, Y. Doi, and A. J. Sievers, Supertransmission channel for an intrinsic localized mode in a one-dimensional nonlinear physical lattice, Chaos 25, 103122 (2015).

[34] D. P. Snowden-Ifft, E. S. Freeman, and P. B. Price, Limits on Dark Matter Using Ancient Mica, Phys. Rev. Lett. 74, 4133 (1995).

[35] E. A. Baltz, A. J. Westphal, and D. P. Snowden-Ifft, Probing the structure of the cold dark matter halo using ancient mica, Phys. Rev. D 59, 023510 (1998).

[36] J. F. R. Archilla, J. Cuevas, M. D. Alba, M. Naranjo, and J. M. Trillo, Discrete breathers for understanding reconstructive mineral processes at low temperatures, J. Phys. Chem. B 110, 24112 (2006). 
[37] J. F. R. Archilla, J. Cuevas, and F. R. Romero, Effect of breather existence on reconstructive transformations in mica muscovite, AIP Conf. Proc. 982, 788 (2008).

[38] V. I. Dubinko, P. A. Selyshchev, and J. F. R. Archilla, Reactionrate theory with account of the crystal anharmonicity, Phys. Rev. E 83, 041124 (2011).

[39] H. G. Hansma, Possible origin of life between mica sheets, J. Theor. Bio. 266, 175 (2010).

[40] H. G. Hansma, Possible origin of life between mica sheets: Does life imitate mica? J. Biomol. Struct. Dyn. 31, 888 (2013).

[41] A. G. Cairns-Smith, Chemistry and the missing era of evolution, Chem. Eur. J. 14, 3830 (2008).

[42] S. S. Kim, T. V Khai, V. Kulish, Y.-H. Kim, H. G. Na, A. Katoch, M. Osada, P. Wu, and $\mathrm{H}$. Kim, Tunable bandgap narrowing induced by controlled molecular thickness in 2D mica nanosheets, Chem. Mater. 27, 4222 (2015).

[43] J. Biersack, J. P. Ziegler, and M. D. Ziegler, SRIM-The Stopping and Range of Ions in Matter (J. P. Ziegler, Chester, MD, 2008).

[44] O. Gedeon, J. Machácek, and M. Liška, Static energy hypersurface mapping of potassium cations in potassium silicate glasses, Phys. Chem. Glasses 43, 241 (2002).

[45] M. Diaz, V. C. Farmer, and R. Prost, Characterization and assignment of far infrared absorption bands of $\mathrm{K}^{+}$in muscovite, Clays Clay Miner. 48, 433 (2000).

[46] D. R. Collins and C. R. A. Catlow, Computer simulation of structures and cohesive properties of micas, Am. Mineral. 77, 1172 (1992).

[47] R. S. MacKay and S. Aubry, Proof of existence of breathers for time-reversible or Hamiltonian networks of weakly coupled oscillators, Nonlinearity 7, 1623 (1994).

[48] S. Aubry, Breathers in nonlinear lattices: Existence, linear stability and quantization, Physica D (Amsterdam) 103, 201 (1997).

[49] J. F. R. Archilla, J. Cuevas, B. Sanchez-Rey, and A. Alvarez, Demonstration of the stability or instability of multibreathers at low coupling, Physica D (Amsterdam) 180, 235 (2003).

[50] G. James, Centre manifold reduction for quasilinear discrete systems, J. Nonlinear Sci. 13, 27 (2003).
[51] B. Sanchez-Rey, G. James, J. Cuevas, and J. F. R. Archilla, Bright and dark breathers in Fermi-Pasta-Ulam lattices, Phys. Rev. B 70, 014301 (2004).

[52] M. Ibañes, J. M. Sancho, and G. P. Tsironis, Dynamical properties of discrete breathers in curved chains with first and second neighbor interactions, Phy. Rev. E 65, 041902 (2002).

[53] J. F. R. Archilla, P. L. Christiansen, S. F. Mingaleev, and Y. B. Gaididei, Numerical study of breathers in a bent chain of oscillators with long-range interaction, J. Phys. A 34, 6363 (2001).

[54] D. Chen, S. Aubry, and G. P. Tsironis, Breather Mobility in Discrete $\phi^{4}$ Nonlinear Lattices, Phys. Rev. Lett. 77, 4776 (1996).

[55] A. Alvarez, F. R. Romero, J. Cuevas, and J. F. R. Archilla, Discrete moving breather collisions in a Klein-Gordon chain of oscillators, Phys. Lett. A 372, 1256 (2008).

[56] M. Johansson and P. Jason, Breather mobility and the PeierlsNabarro potential: Brief review and recent progress, in Quodons in Mica: Nonlinear Localized Travelling Excitations in Crystals, Springer Series in Materials Science, Vol. 221, edited by J. F. R. Archilla et al. (Springer, Cham, 2015), pp. 147-178.

[57] K. Yoshimura and Y. Doi, Moving discrete breathers in nonlinear lattice: Resonance and stability, Wave Motion 45, 83 (2007).

[58] G. James and Y. Sire, Travelling breathers with exponentially small tails in a chain of nonlinear oscillators, Commun. Math. Phys. 257, 51 (2005).

[59] J. Cuevas-Maraver, P. G. Kevrekidis, A. Vainchtein, and H. $\mathrm{Xu}$, Unifying perspective: Solitary traveling waves as discrete breathers in Hamiltonian lattices and energy criteria for their stability, Phys. Rev. E 96, 032214 (2017).

[60] Y. Doi and K. Yoshimura, Symmetric Potential Lattice and Smooth Propagation of Tail-Free Discrete Breathers, Phys. Rev. Lett. 117, 014101 (2016).

[61] J. P. Boyd, A numerical calculation of a weakly nonlocal solitary wave: The $\phi^{4}$ breather, Nonlinearity 3, 177 (1990).

[62] J. Gomez-Gardeñes, F. Falo, and L. M. Floría, Mobile localization in nonlinear Schrödinger lattices, Phys. Lett. A 332, 213 (2004).

[63] S. Aubry, Discrete breathers: Localization and transfer of energy in discrete Hamiltonian nonlinear systems, Physica D (Amsterdam) 216, 1 (2006). 